\title{
Multiple decomposability of probabilities on contractible locally compact groups
}

\author{
Wilfried Hazod, Katrin Kosfeld \\ Preprint 2011-04 \\ Februar 2011
}

Fakultät für Mathematik

Technische Universität Dortmund

Vogelpothsweg 87

44227 Dortmund

$\underline{\text { tu-dortmund.de/MathPreprints }}$ 



\title{
MULTIPLE DECOMPOSABILITY OF PROBABILITIES ON CONTRACTIBLE LOCALLY COMPACT GROUPS
}

\author{
WILFRIED HAZOD, KATRIN KOSFELD
}

\begin{abstract}
Operator decomposable probabilities on vector spaces - generalizing (semi-)stable and self-decomposable laws - are well known. More specific concepts, multiple operator decomposable laws, generalizing the nested Urbanik classes in the case of selfdecomposability, were investigated in fundamental papers by Maejima et al. [16], [17] resp. by Maejima and R. Shah [18] for real resp. $p$-adic vector spaces. For locally compact groups, decomposability properties were studied by R. Shah [27], Raja [23], see also [6]. Here we are concerned with multiple decomposability on locally compact groups and generalize - as far as possible - the results in [16], [18]. In fact, as it turned out that contraction properties play an essential role, hence throughout we concentrate on contractible locally compact groups.
\end{abstract}

\section{INTRODUCTION}

On vector spaces, operator-decomposability of probabilities - a common setup for operator-semi-stable, stable and self-decomposable laws - were the subject of various investigations. In analogy to the Urbanik classes in the case of self-decomposability, nested classes of multiple operator decomposable laws and their properties were investigated. For the background the reader is referred e.g., to [12], [31], [32] for finite and infinite dimensional real vector spaces, and [16] and the references therein for multiple decomposability. In case of locally compact groups we mention R. Shah [27], Raja [23], see also [6], and for multiple decomposability on $p$-adic groups and vector spaces, [18] and the literature mentioned there. (For some aspects of multiple self-decomposability, i.e., decomposability w.r.t. a continuous one-parameter group of automorphisms, on groups see e.g., [4].)

Let $\mathbb{G}$ be a locally compact group and $\tau \in \operatorname{Aut}(\mathbb{G})$. A probability $\mu \in \mathcal{M}^{1}(\mathbb{G})$ is called $\tau$-decomposable - in symbols: $\mu \in L_{0}(\tau)$ - if there exists a cofactor $\nu \in \mathcal{M}^{1}(\mathbb{G})$ such that $\mu=\nu \star \tau(\mu)$. Put $L_{-1}(\tau):=$ $\mathcal{M}^{1}(\mathbb{G}) . \quad \mu$ is called $n$-times $\tau$-decomposable, $n \geq 0$, - in symbols: $\mu \in L_{n}(\tau)$ - if $\mu$ is $\tau$-decomposable with a cofactor $\nu \in L_{n-1}(\tau)$. (For details cf. Definition 4.1 below.)

A continuous convolution semigroup $\left(\mu_{t}\right)_{t \geq 0}$, of probabilities is $\tau$ decomposable if for all $t>0, \mu_{t}$ is $\tau$-decomposable with cofactor $\nu_{t}$, where the cofactors $\left(\nu_{t}\right)_{t \geq 0}$ form a continuous convolution semigroup. Analogously, $\left(\mu_{t}\right)_{t \geq 0}$ is $n$-times $\tau$-decomposable if it is $\tau$-decomposable and the cofactor semigroup is $(n-1)$-times $\tau$-decomposable.

Date: 30.1 .11 . 
This motivates the following definition: Let $\mathcal{A}$ denote the generating functional of $\left(\mu_{t}\right)_{t \geq 0}, \mathcal{A}=\left.\frac{\mathrm{d}^{+}}{\mathrm{d} t}\right|_{t=0}\left\langle\mu_{t}, f\right\rangle$, evaluated for $f \in \mathcal{D}(\mathbb{G})$, the Bruhat-Schwartz test function space. Let $\mathcal{G} \mathcal{F}(\mathbb{G})$ denote the cone of generating functionals. $\mathcal{A}$ is $\tau$-decomposable - in symbols: $\mathcal{A} \in \Lambda_{0}(\tau)$ - if for some generating functional $\mathcal{A}^{(1)}$ we have $\mathcal{A}=\mathcal{A}^{(1)}+\tau(\mathcal{A})$. Put $\Lambda_{-1}(\tau):=\mathcal{G F}(\mathbb{G})$. Analogously, $\mathcal{A}$ is $n$-times $\tau$-decomposable $\left(n \in \mathbb{Z}_{+}\right)$- in symbols: $\mathcal{A} \in \Lambda_{n}(\tau)-$ if $\mathcal{A} \in \Lambda_{0}(\tau), \mathcal{A}=\mathcal{A}^{(n)}+\tau(\mathcal{A})$, with $\mathcal{A}^{(n)} \in \Lambda_{n-1}(\tau)$. Hence there exist generating functionals $\mathcal{A}^{(i)}$ such that $\mathcal{A}^{(i)}=\mathcal{A}^{(i-1)}+\tau\left(\mathcal{A}^{(i)}\right)$ for $1 \leq i \leq n+1$ with $\mathcal{A}^{(n+1)}:=\mathcal{A}$. Thus $\mathcal{A}=\mathcal{A}^{(0)}+\sum_{1}^{n+1} \tau\left(\mathcal{A}^{(i)}\right)$. (For more details cf. Definition 5.5.)

Recall that for non-Abelian groups, generating functionals of continuous convolution semigroups play the role of logarithms of Fourier transforms, the second characteristic functions. For details the reader is referred to, e.g., [10], Ch IV, § 2.0, [7], [29], [3].

Throughout we have to suppose that $\mathbb{G}$ is contractible and $\tau \in$ $\operatorname{Aut}(\mathbb{G})$ is contracting, a natural condition. In complete analogy to the vector space case, we prove that $n$-times $\tau$-decomposable laws are representable as infinite convolution products, $\mu=\underset{j \geq 0}{\star} \tau^{j}(\nu)($ for $n=0)$ resp. $\mu=\underset{\substack{j_{n} \geq 0 \\ \star}}{\star}\left(\cdots\left(\underset{\substack{\star \\ j_{0} \geq 0}}{\star \tau_{0}^{n} j_{i}}(\nu)\right)\right)$ (for $\left.n \geq 1\right)$. (Cf. Proposition 4.2). Under commutativity assumptions - and if a technical condition is satisfied (trivially satisfied e.g. in the case of homogeneous groups) - we obtain, re-arranging the iterated convolution products: $\mu$ is $n$ times $\tau$-decomposable iff $\mu=\underset{k \geq 0}{\star} \tau^{k}\left(\nu^{\left(\begin{array}{c}n+k \\ n\end{array}\right)}\right)$, and this is the case iff $\nu$ possesses logarithmic moments of order $n+1$.

In case of continuously embeddable probabilities we obtain, again in analogy to the vector space situation - at least for connected resp. totally disconnected contractible groups - a characterization of multiple $\tau$-decomposable generating functionals of continuous convolution semigroups which corresponds to the characterization of the Lévy-Khinchin form in the case of real resp. $p$-adic vector spaces obtained in [16] resp. [18].

Most of the results are part of the second named author's thesis [13].

The paper is organized as follows: First, in Section 1 we state the main results, Theorems 1.1 and 1.2. For their proofs we need several tools which are of independent interest. These tools, group norms and automorphism norms, resp. convergence of infinite convolution products, are collected in the following Sections 2 resp. 3. The proofs of the main results, and some slightly more general results follow in Sections 4 and 5 in a sequence of propositions. In an appendix, we discuss briefly the possibility of analogous investigations for certain classes of hypergroups.

\section{MAin Results}

Throughout let $\mathbb{G}$ denote a contractible locally compact group with contracting automorphism $\tau \in \operatorname{Aut}(\mathbb{G}) . \quad \mu$ has a finite logarithmic 
moment of order $n$ (in symbols, $\mu \in \mathcal{P}_{\log ^{n}}$ ) if for a random variable $X$ with distribution $\mu, \mathbb{E}\left(\left(\log _{+}(|X|)\right)^{n}\right)=\int_{\mathbb{G}}\left(\log _{+}|x|\right)^{n} \mathrm{~d} \mu(x)<\infty$, where $|\cdot|$ denotes a group norm on $\mathbb{G}$. (Cf. Definition 2.1). As afore mentioned, $\mu$ is $n$-times $\tau$-decomposable iff $\mu$ has a representation as $(n+1)$-times iterated convolution product. To be allowed to re-arrange convolution products (in case $n>0$ ) we define $\mathcal{S} \subseteq \mathcal{M}^{1}(\mathbb{G})$ to be a commutative sub-semigroup with $\tau(\mathcal{S}) \subseteq \mathcal{S}$ and $\nu \in \mathcal{S}$.

Theorem 1.1. Let $\mu, \nu \in \mathcal{S}, n \in \mathbb{Z}_{+}$.

a) $\mu \in \mathcal{M}^{1}(\mathbb{G})$ is n-times $\tau$-decomposable iff

$$
\mu=\underset{j_{n} \geq 0}{\star}\left(\underset{j_{n-1} \geq 0}{\star} \cdots\left(\underset{j_{0} \geq 0}{\star} \tau^{\sum_{1}^{n} j_{i}}(\nu)\right) \cdots\right)
$$

And if the right invariance group is trivial, $H_{r}(\mu):=\left\{x: \mu \star \varepsilon_{x}=\mu\right\}=$ $\{e\}$, then

$$
\mu=\underset{j=0}{\star} \tau^{j}\left(\nu\left(\begin{array}{c}
n+j \\
n
\end{array}\right)\right)
$$

b1) If $\nu \in \mathcal{P}_{\log _{+}^{n+1}}$, the convolution products (1.1), (1.2) and all rest products $\lambda_{k}:=\underset{j=k+1}{\star} \tau^{j}\left(\nu^{\left(\begin{array}{c}n+j \\ n\end{array}\right)}\right), k \in \mathbb{Z}_{+}$, converge and $\lambda_{k} \stackrel{k \rightarrow \infty}{\rightarrow} \varepsilon_{e}$.

b2) Conversely, assume that the products (1.1) or (1.2) are converging, and again $H_{r}(\mu)=\{e\}$, then $\nu \in \mathcal{P}_{\text {log }_{+}^{n+1}}$.

If $\mathbb{G}$ is a homogeneous group, hence aperiodic, all invariance groups are trivial, and in case $n \geq 0$, the condition $H_{r}(\mu)=\{e\}$ is trivially satisfied. And also for general locally compact contractible groups, for $n=0$, the condition $H_{r}(\mu)=\{e\}$ is superfluous. (Cf. Proposition 3.9).

Let $\mu$ be embedded into a continuous convolution semigroup $\left(\mu_{t}\right)$, and let $\mathcal{A}$ denote the generating functional. Let (multiple) $\tau$-decomposability of continuous convolution semigroups resp. of generating functionals be defined as afore. In this case we obtain

Theorem 1.2. a) Let $\mathbb{G}$ be a connected contractible group, hence a homogeneous group, and let $\mathcal{G} \cong \mathbb{R}^{d}$ denote the Lie algebra. Let $\tau \in \operatorname{Aut}(\mathbb{G})$ and let $\stackrel{\circ}{\tau} \in \operatorname{Aut}(\mathcal{G}) \subseteq \mathrm{GL}\left(\mathbb{R}^{d}\right)$ denote the differential. Hence $\exp ^{-1} \circ \tau=\stackrel{\circ}{\tau} \circ \exp ^{-1}$. Let $\stackrel{\circ}{\mathcal{A}}$ denote the image of $\mathcal{A}$ under $\exp ^{-1}$. Then we have:

$\mathcal{A}$ is $n$-times $\tau$-decomposable (on $\mathbb{G}$ ) iff $\stackrel{\circ}{\mathcal{A}}$ is $n$-times $\tau$-decomposable (on the vector space $\mathcal{G}$ ). Equivalently, if the continuous convolution semigroup $\left(\lambda_{t}\right) \subseteq \mathcal{M}^{1}(\mathcal{G})$ generated by $\stackrel{\circ}{\mathcal{A}}$ is $n$-times $\stackrel{\circ}{\tau}$-decomposable.

This is the case iff the Lévy-Khinchin representation of $\stackrel{\circ}{\mathcal{A}}$ has the form given in [16], Theorem 3.1.

b) Let $\mathbb{G}$ be a contractible totally disconnected locally compact group. Then there exist $p \in \mathbb{N}, p>1$ (not necessarily a prime power), a homeomorphism $\psi: \mathbb{G} \rightarrow \mathcal{G}:=\mathbb{Q}_{p}$, the additive group of $p$-adic numbers, and $\stackrel{\circ}{\tau} \in \operatorname{Aut}\left(\mathbb{Q}_{p}\right)$, such that $\psi \circ \tau=\stackrel{\circ}{\tau} \circ \psi$. Let again for a generating functional $\mathcal{A}, \stackrel{\mathcal{A}}{:}=\psi(\mathcal{A})$ denote the image under $\psi$. Then we have: 
$\mathcal{A}$ is $n$-times $\tau$-decomposable (on $\mathbb{G}$ ) iff $\stackrel{\circ}{\mathcal{A}}$ is $n$-times $\stackrel{\circ}{\tau}$-decomposable (on $\mathcal{G})$. Equivalently, if the continuous convolution semigroup $\left(\lambda_{t}\right) \subseteq \mathcal{M}^{1}(\mathcal{G})$ generated by $\stackrel{\circ}{\mathcal{A}}$ is n-times $\stackrel{\circ}{\tau}$-decomposable.

This is the case iff the Lévy-Khinchin representation of $\stackrel{\circ}{\mathcal{A}}$ on $\mathbb{Q}_{p}$ has the form given in [18], Theorems 4.3, 5.2.

\section{GRoup NORMS AND AUTOMORPHISM NORMS ON CONTRACTIBLE GROUPS}

Let $\mathbb{G}$ denote a locally compact group, Aut $(\mathbb{G})$ the group of topological automorphisms endowed with the topology of uniform convergence on compact subsets.

Definition 2.1. A group norm on $\mathbb{G}$ is a continuous sub-additive functional $|\cdot|: \mathbb{G} \rightarrow \mathbb{R}_{+}$such that

(i) $|x|=\left|x^{-1}\right|$ for all $x \in \mathbb{G}$

(ii) $|x|=0$ iff $x=e$

(iii) $x_{n} \rightarrow e$ iff $\left|x_{n}\right| \rightarrow 0$

Thus $d(x, y):=\left|x^{-1} y\right|$ defines a right-invariant metric generating the topology of $\mathbb{G}$.

Remark 2.2. In the case of contractible locally compact groups we shall show that always group norms exist (see Proposition 2.10). Note that for homogeneous groups (homogeneous) norms are usually defined by a weaker condition $|x y| \leq C(|x|+|y|)$ for some $C \geq 1$. However it is known that sub-additive homogeneous norms, i.e., norms with $C=1$, exist (cf. [8]). Hence here we restrict to sub-additive functionals.

Note furthermore, that group norms are frequently called pseudonorms (generating the topology of $\mathbb{G}$ ), cf. e.g., [33].

Obviously we have: Let $|\cdot|_{i}, i=1,2$, and $|\cdot|$ be group norms. Then the following functionals are also group norms:

1) $|\cdot|_{*}:=\max _{i=1,2}|\cdot|_{i}$ 2) $\quad|\cdot|_{0}:=|\cdot|_{1}+|\cdot|_{2}$

3) $|\cdot|_{\sigma}:=|\sigma(\cdot)|$ for $\sigma \in \operatorname{Aut}(\mathbb{G})$ and 4) $\log (|\cdot|+1)$.

In the sequel we make use of the following examples:

Example 2.3. Let $\mathbb{G}$ be a connected contractible group, therefore a homogeneous group. In particular, $\mathbb{G}$ is a simply connected nilpotent Lie group admitting a continuous one-parameter group of dilations $\left(\delta_{t}\right)_{t>0} \subseteq \operatorname{Aut}(\mathbb{G})$, such that $\delta_{t} \delta_{s}=\delta_{t \cdot s}$ and $\delta_{t}(x) \rightarrow e, t \rightarrow 0$, for all $x \in \mathbb{G}$. Then there exists a group norm satisfying $\left|\delta_{t}(x)\right|=t \cdot|x|$ for $t>0, x \in \mathbb{G}$. Thus $\mathbb{G} \backslash\{e\} \cong \mathbb{R}_{+}^{\times} \otimes\{x:|x|=1\}$. Such a norm - a subadditive homogeneous norm - will be called adapted to the dilations.

Any two homogeneous norms adapted to $\left(\delta_{t}\right)$ are equivalent. For details see, e.g., [7], 2.9., and the literature mentioned there.

Example 2.4. Let $\mathbb{G}$ be totally disconnected and assume that there exist compact open subgroups $U_{n}, n \in \mathbb{Z}$ such that $U_{n+1} \triangleleft U_{n}, n \in \mathbb{Z}$, $\bigcup U_{n}=\mathbb{G}, \cap U_{n}=\{e\} . \quad\left(\left(U_{n}\right)\right.$ is called filtration by compact open subgroups.) Fix $0<\alpha<1$. Define $|x|:=|x|_{\alpha}:=\alpha^{k}$ if $x \in U_{k} \backslash U_{k+1}$, 
and $|e|:=0$. Then $|\cdot|$ is a group norm, adapted to $\left(U_{n}\right)$. 【 In fact, let e.g., $k \leq \ell, x \in U_{k}, y \in U_{\ell}$. Then $x y \in U_{k} U_{\ell} \subseteq U_{k}$. Hence $|x y| \leq \alpha^{k}$. I.e., $|x y| \leq \max \{|x|,|y|\} \leq|x|+|y|$. ] The rest assertions are immediately verified.

Finite dimensional vector spaces are (commutative) homogeneous groups, with homothetical transformations $H_{t}: x \mapsto t \cdot x$ as dilations. And vector space norms are homogeneous norms. As on vector spaces, homogeneous norms on a homogeneous group (w.r.t. fixed dilations) are equivalent. In contrast, this is not the case for group norms (adapted to $\tau$ ) as the following example shows:

Example 2.5. Let as in Examples 2.4 resp. 2.8, $\left(U_{n}\right)_{n \in \mathbb{Z}}$ be a filtration of $\mathbb{G}$ with compact neighbourhoods of e, i.e., $U_{n} \supseteq U_{n+1}, \bigcup U_{n}=$ $\mathbb{G}, \bigcap U_{n}=\{e\}$. Let $|\cdot|$ be a group norm, and assume for some $\alpha \in(0,1)$ that $\alpha^{n+1} \leq|x| \leq \alpha^{n}$ for $x \in U_{n} \backslash U_{(n+1)}$. Consider the group norm $|x|_{1}:=\log (|x|+1)$. For $x_{n} \in U_{-(n+1)} \backslash U_{-n}, n \in \mathbb{N}$, we have $\left|x_{n}\right|,\left|x_{n}\right|_{1} \rightarrow \infty$, but $\left|x_{n}\right|_{1} /\left|x_{n}\right| \geq \alpha^{-n+1} / \log \left(1+\alpha^{-n}\right) \rightarrow \infty$. Hence the norms are not equivalent.

As easily verified, such filtrations exist for the groups considered in Examples 2.3 and 2.4 .

Definition 2.6. An automorphism norm corresponding to a group norm $|\cdot|$ is a sub-multiplicative functional $\|\cdot\|: \operatorname{Aut}(\mathbb{G}) \rightarrow \mathbb{R}_{+}$satisfying $|\sigma(x)| \leq\|\sigma\| \cdot|x|(x \in \mathbb{G}, \sigma \in \operatorname{Aut}(\mathbb{G}))$.

$\|\sigma\|_{0}:=\sup _{x \neq e}|\sigma(x)| /|x|$ is obviously an automorphism norm. It will be called canonical automorphism norm (w.r.t|-|). If not otherwise defined, $\|\cdot\|$ will always denote a canonical automorphism norm.

$\tau \in \operatorname{Aut}(\mathbb{G})$ is called contracting if $\tau^{n}(x) \stackrel{n \rightarrow \infty}{\rightarrow} e$ for all $x$. As well known, if $\tau$ is contracting then it is compactly contracting, i.e., for all compact $K \subseteq \mathbb{G}$ we have $\tau^{n}(K) \rightarrow\{e\}$. (Cf. [30], Lemma 1.4 (iv), [35]).

We continue the previous Examples 2.3, 2.4:

Example 2.7. Let $\mathbb{G}$ be a contractible connected group as in example 2.3 with contracting $\tau \in \operatorname{Aut}(\mathbb{G})$. Then there exist a group of dilations $\left(\delta_{t}\right)$ with $\tau \delta_{t}=\delta_{t} \tau$ for all $t$. (Cf. e.g., [7], Remark 2.1.13 a)). Let $|\cdot|$ denote a (sub-additive homogeneous) norm adapted to the dilations. For short, adapted to $\tau$. Then, as easily verified, for all automorphisms $\sigma \in \operatorname{Cent}\left(\left(\delta_{t}\right)\right)$, in particular for $\sigma=\tau$, we have for the canonical norm:

1) $\|\sigma\|=\sup _{x \neq e}|\sigma(x)| /|x|=\max _{|x|=1}|\sigma(x)|=\max _{|x| \leq 1}|\sigma(x)|$

2) $\tau$ is (compactly) contracting, hence $\tau^{n}\{x:|x|=1\} \rightarrow\{e\}$.

3) Therefore $\left\|\tau^{m}\right\| \rightarrow 0$, hence there exists $m_{0} \in \mathbb{N}$ with $\left\|\tau^{m}\right\|<1$ for all $m \geq m_{0}$.

4) and $\left\|\tau^{-1}\right\|>1$.

Example 2.8. Let, as in example 2.4, $\mathbb{G}$ be totally disconnected and contractible with contracting $\tau \in \operatorname{Aut}(\mathbb{G})$. There exists a filtration $\left(U_{n}\right)$ by open compact subgroups such that in addition $\tau U_{n}=U_{n+1}$, $n \in \mathbb{Z}$. We define for $0<\alpha<1$ the norm as in example 2.4. Then by 
construction, $\left|\tau^{n}(x)\right|=\alpha^{n} \cdot|x|, x \in \mathbb{G}$. . Therefore, for the canonical automorphism norm, we obtain $\left\|\tau^{n}\right\|=\alpha^{n}$. In particular, $\|\tau\|=\alpha<1$ and $\left\|\tau^{-1}\right\|=\alpha^{-1}>1$.

Lemma 2.9. Let $\mathbb{G}$ be a contractible locally compact group with contracting $\tau \in \operatorname{Aut}(\mathbb{G})$. Let $|\cdot|$ be a group norm and assume for some $m_{0} \geq 1,\left\|\tau^{m}\right\|<1$ for $m \geq m_{0}$. Then there exist $0<r<R<1$, $m_{1} \geq m_{0}$, such that for $N \geq m_{1}$

$$
\begin{array}{r}
r^{N} \cdot|x| \leq\left|\tau^{N}(x)\right| \leq R^{N} \cdot|x| \\
R^{-N} \cdot|x| \leq\left|\tau^{-N}(x)\right| \leq r^{-N} \cdot|x|
\end{array}
$$

Proof. Let $N \geq m_{0}, N=k \cdot m_{0}+j, 0 \leq j<m_{0}$. Then

$$
\left|\tau^{N}(x)\right| \leq\left\|\tau^{m_{0}}||^{k} \cdot\left|\tau^{j}(x)\right| \leq\left(\| \tau^{m_{0}}||^{1 / m_{0}}\right)^{k} \cdot\right\| \tau^{j} \| \cdot|x| \leq C \cdot R_{0}^{N} \cdot|x|
$$

for $R_{0}:=\left\|\tau^{m_{0}}\right\|^{1 / m_{0}}<1$ and $C \geq \max _{0 \leq j<m_{0}}\left(\left\|\tau^{j}\right\| R_{0}^{-j}\right)(\geq 1)$.

Hence $\left|\tau^{N}(x)\right| \leq\left(C^{1 / N} \cdot R_{0}\right)^{N}|x|$. For sufficiently large $N \geq m_{1}(\geq$ $\left.m_{0}\right)$ we have $C^{1 / N} \cdot R_{0} \leq C^{1 / m_{1}} \cdot R_{0}=: R_{1}<1$. Thus the right side of (2.1) holds for $1>R \geq R_{1}$. The left side, as immediately seen, is valid for $0<r \leq r_{1}:=\left\|\tau^{-1}\right\|^{-1}$ and all $N \in \mathbb{Z}_{+}$.

Again the right hand side of (2.2) follows immediately for all $r^{-1} \geq$ $r_{1}^{-1}:=\left\|\tau^{-1}\right\|>1$ and and all $N \in \mathbb{Z}_{+}$. And almost verbatim as in the proof of (2.1), the left hand follows for $1<R^{-1} \leq R_{1}^{-1}$.

Whence the assertion follows.

According to Examples 2.7 and 2.8 the assumptions of Lemma 2.9 are satisfied for connected resp. totally disconnected contractible groups. In that cases the growth conditions $(2.1)$ and (2.2) hold. Now let $\mathbb{G}$ be a contractible locally compact group with contracting $\tau \in \operatorname{Aut}(\mathbb{G})$. Then $\mathbb{G}=\mathcal{N} \otimes \mathbb{D}$, where $\mathcal{N}$ is connected and $\mathbb{D}$ totally disconnected with contracting $\rho \in \operatorname{Aut}(\mathcal{N})$ and $\sigma \in \operatorname{Aut}(\mathbb{D})$ respectively, such that $\tau=\rho \otimes \sigma . \quad$ (Cf. [30], Proposition 4.2, Corollary 4.3, [7], 3.1.12). According to the examples 2.7 and 2.8 there exist group-norms $|\cdot|_{\mathcal{N}}$ and $|\cdot|_{\mathbb{D}}$ on $\mathcal{N}$ and $\mathbb{D}$ adapted to $\rho$ and $\sigma$ respectively.

Then $|\cdot|$, defined by $|(x, y)|:=|x|_{\mathcal{N}}+|y|_{\mathbb{D}}$, for $(x, y) \in \mathcal{N} \otimes \mathbb{D}=\mathbb{G}$, is a group norm on $\mathbb{G}$, adapted to $\tau$. Hence we have

Proposition 2.10. Let $\mathbb{G}$ be a contractible locally compact group with contracting $\tau \in \operatorname{Aut}(\mathbb{G})$. There exists a group norm $|\cdot|$ on $\mathbb{G}$, with corresponding automorphism norm satisfying the growth conditions (2.1) and (2.2).

Proof. As mentioned above, $\mathbb{G}$ splits as a direct product $\mathbb{G}=\mathcal{N} \otimes \mathbb{D}$ and also $\tau=\rho \otimes \sigma$. $\rho$ and $\sigma$ are both contracting on $\mathcal{N}$ and $\mathbb{D}$ respectively and satisfy the growth conditions (2.1) and (2.2) for $1>R \geq R_{i}$ and $0<r \leq r_{i} \leq R_{i}, i=1,2$. In case of $\mathcal{N},(2.1)$ and (2.2) hold for $R \geq R_{1}$ and $N \geq m_{1} \geq 0$. (Cf. Examples 2.7 and 2.8.)

With the group norm on $\mathbb{G}$ defined afore, as immediately verified, $\tau$ satisfies (2.1) and (2.2), with $1>R \geq \max \left\{R_{1}, R_{2}\right\}, 0<r \leq$ $\min \left\{r_{1}, r_{2}\right\}$ and $N \geq m_{1}$.

For later use (in Section 5) we note: 
Proposition 2.11. a) Let $\mathbb{G}$ be a connected contractible group with contracting $\tau \in \operatorname{Aut}(\mathbb{G})$, therefore a (homogeneous, hence nilpotent and simply connected) Lie group of dimension $d$. Let $\mathcal{G} \cong \mathbb{R}^{d}$ denote the Lie algebra and let $\stackrel{\circ}{\tau} \in \operatorname{Aut}(\mathcal{G}) \subseteq \mathrm{GL}\left(\mathbb{R}^{d}\right)$ denote the differential of $\tau$. $\exp ^{-1}: \mathbb{G} \rightarrow \mathcal{G}$ is a $C^{\infty}$-homeomorphism satisfying

$$
\exp ^{-1} \circ \tau=\stackrel{\circ}{\tau} \circ \exp ^{-1}
$$

b) Let $\mathbb{G}$ be a totally disconnected contractible group with contracting $\tau \in \operatorname{Aut}(\mathbb{G})$. Then there exists $p \in \mathbb{N} \backslash\{1\}, \stackrel{\circ}{\tau} \in \operatorname{Aut}(\mathcal{G})$ and a homeomorphism $\psi: \mathbb{G} \rightarrow \mathcal{G} \cong \mathbb{Q}_{p}$ satisfying

$$
\psi \circ \tau=\stackrel{\circ}{\tau} \circ \psi .
$$

Here $\mathcal{G}=\mathbb{Q}_{p}$ denotes the additive group of $p$-adic numbers. ( $p$ is not assumed to be a prime power here.)

Proof. a) The assertion is obvious since $\mathbb{G}$ is a simply connected nilpotent Lie group, hence homeomorphic to $\mathcal{G}$.

b) Let $\left(U_{n}\right)$ be a filtration by open compact subgroups adapted to $\tau$. Put $p:=\operatorname{ord}\left(U_{0} / U_{1}\right)$. [30], Proposition 4.2, Corollary 4.3, (resp. [7], 3.1.8-3.1.10) contains explicit constructions of the homeomorphism $\psi$ and of $\stackrel{\circ}{\tau}$. In fact, the construction there is the following:

Let $\mathbb{Z}_{p}:=\{0, \ldots, p-1\}$ and let $\mathcal{P} \subseteq \bigotimes_{\mathbb{Z}} \mathbb{Z}_{p}$ denote the set of sequences $\left\{\vec{x}:=(x(k))_{k \in \mathbb{Z}}: x(k) \in \mathbb{Z}_{p}, x(k)=0 \forall k \leq K(\vec{x})\right\}$. Every subset $\Lambda_{k}:=\{\vec{x}: x(j)=0 \forall j \leq k\}$ is compact w.r.t. the product topology. And endowed with the topology that makes all cosets $y \cdot \Lambda_{k}:=$ $\left\{y(k) x(k): \vec{x} \in \Lambda_{k}\right\}$ compact open, $\mathcal{P}$ is a totally disconnected space. If we define the group operation coordinate-wise, $\mathcal{P}$ becomes a locally compact group, which will be called $\Lambda$. This group is contractible, since the shift $\sigma$, defined by $(\sigma(\vec{x}))(k):=x(k+1)$, is a contracting automorphism with the property $\sigma\left(\Lambda_{n}\right)=\Lambda_{n+1}$. Furthermore, there exists a homeomorphism $\phi_{1}: \mathbb{G} \rightarrow \Lambda$ such that $\phi_{1} \circ \tau=\sigma \circ \phi_{1}$, and $\phi_{1}\left(U_{n}\right)=\Lambda_{n}$ for all $n$.

Let $\mathbb{Q}_{p}$ denote the additive group of the ring of $p$-adic numbers, let $H_{p}: r \mapsto p \cdot r$ denote the homothetical transformation on $\mathbb{Q}_{p}$. We have $H_{p} \in \operatorname{Aut}\left(\mathbb{Q}_{p}\right)$, in fact, $H_{p}$ is contracting. If $\Delta_{0}$ denotes the $p$ adic integers, then $\Delta_{k}:=H_{p^{k}}\left(\Delta_{0}\right), k \in \mathbb{Z}$, is a filtration of $\mathbb{Q}_{p}$ with $p=\operatorname{ord}\left(\Delta_{k} / \Delta_{k+1}\right)$. (Note that $\mathbb{Q}_{p}$ may be represented as the space $\mathcal{P}$ defined above, with a different algebraic structure. See e.g., [9], 10.2 - 10.10. $H_{p}$ operates as shift on this product space.) Hence there exists a homeomorphism $\phi_{2}: \mathbb{Q}_{p} \rightarrow \Lambda$ such that $\phi_{2} \circ H_{p}=\sigma \circ \phi_{2}$ and $\phi_{2}\left(\Delta_{k}\right)=\Lambda_{k}$ for all $k$.

Hence with the composition $\psi: \mathbb{G} \stackrel{\phi_{1}}{\longrightarrow} \Lambda \stackrel{\phi_{2}^{-1}}{\longrightarrow} \mathbb{Q}_{p}$ and $\stackrel{\circ}{\tau}:=H_{p}$ the assertion follows. [ In fact, in [30], 3.7, [7], 3.2.8, it is supposed that $p$ is a prime power, hence $\mathbb{Q}_{p}$ a field. But the construction does not depend on this assumption. ] 


\section{Convergence of Convolution products on CONTRACtible GROUPS}

Recall that for locally compact groups some versions of P. Lévy's equivalence theorem are available. For our purposes we need the following versions:

Proposition 3.1. Let $\mathbb{G}$ be a locally compact group. (W.l.o.g., $\mathbb{G}$ is assumed to be second countable, as random variables are involved).

a) Let $\mathbb{G}$ be aperiodic, i.e., without non-trivial compact subgroups. Then the classical version of the equivalence theorem holds:

If $\nu_{i}, i \geq 0, \nu$, are probabilities and $X_{i}, i \geq 0, Y$ are $\mathbb{G}$-valued random variables with distributions $X_{i}(P)=\nu_{i}, i \geq 0, Y(P)=\nu$. Then the following assertions are equivalent:

(i) $\prod_{i=0}^{N} X_{i}:=X_{0} \ldots X_{N} \stackrel{N \rightarrow \infty}{\rightarrow} Y$ P-a.s.

(ii) $\prod_{i=0}^{N} X_{i} \stackrel{N \rightarrow \infty}{\rightarrow} Y$ P-stochastically

(iii) $\mu_{N}:=\underset{i=0}{\star} \nu_{i}^{N} \stackrel{N \rightarrow \infty}{\rightarrow} \mu=Y(P)$ weakly

(iii') For all $k \geq-1$ the products $\lambda_{k, N}:=\underset{i=k+1}{\star} \nu_{i}$ are weakly convergent to $\lambda_{k}($ for $N \rightarrow \infty)$ with $\lambda_{-1}=\mu$, and moreover, $\lambda_{k}=\underset{i=k+1}{\star \infty} \nu_{i} \rightarrow$ $\varepsilon_{e}($ for $k \rightarrow \infty)$.

b) If $\mathbb{G}$ is not aperiodic then still ' $(i) \Rightarrow(i i) \Rightarrow\left(i i i^{\prime}\right) \Rightarrow(\text { iii })^{\prime}$ ', and ' $($ iiii' $) \Rightarrow(i)^{\prime}$ hold.

For ' $($ iii $) \Rightarrow(i)$ ' additional conditions are needed:

Let $H_{r}(\lambda):=\left\{x \in \mathbb{G}: \lambda \star \varepsilon_{x}=\lambda\right\}$ denote the right invariance group of $\lambda \in \mathcal{M}^{1}(\mathbb{G})$. If we assume in addition $H_{r}(\mu)=\{e\}$, then $(i i i) \Rightarrow(i)$.

Note that as $\mathbb{G}$ is not supposed to be Abelian we have to define products in a fixed order: $\prod_{0}^{N} x_{i}:=x_{0} x_{1} \cdots x_{N}$ for $x_{i} \in \mathbb{G}$, and analogously $N$

$\star \nu_{i}:=\nu_{0} \star \cdots \nu_{N}$ for probabilities $\nu_{i}$.

0

Proof. For any second countable locally compact group $\mathbb{G}$, the implications ' $(i) \Rightarrow(i i) \Rightarrow(i i i)^{\prime}$ are obvious. To prove ' $(i i) \Rightarrow\left(i i i^{\prime}\right)^{\prime}$ note that a.s. or stochastic convergence of $\prod_{i=0}^{\infty} X_{i}$ imply for any $k \in \mathbb{Z}_{+}$ convergence of $\prod_{i=k}^{\infty} X_{i}$ and moreover, $\prod_{i=k}^{\infty} X_{i} \stackrel{k \rightarrow \infty}{\rightarrow} e$. Thus $\left(i i i^{\prime}\right)$ follows.

Claim: (iii) implies that $\left\{\lambda_{k, n}\right\}_{0 \leq k \leq n}$ is relatively compact. A crucial condition in [10], 2.2.16-2.2.19.

We have for $N, M>0: \mu_{N+M}=\mu_{N} \star \lambda_{N, N+M}$. According to the shift compactness theorem [22], III, theorem 2.1, 2.2, [10], theorem 1.2.21, the set $\left\{\lambda_{N, N+M}\right\}$ is relatively compact. Let $\mathcal{L}$ denote the set of accumulation points (for $N \rightarrow \infty$ ). Let $\lambda \in \mathcal{L}$ and assume, for 
some subsequence, $\lambda_{k_{N}, n_{N}} \rightarrow \lambda$. Then, for $K \in \mathbb{N}, k_{N} \geq K$ we have $\lambda_{K, n_{N}}=\lambda_{K, k_{N}} \star \lambda_{k_{N}, n_{N}}$.

$$
'\left(i i i^{\prime}\right) \Rightarrow(i)^{\prime} \quad \text { By assumption }\left(i i i^{\prime}\right), \lambda_{K, n_{N}} \rightarrow \lambda_{K}:=\underset{j=K+1}{\star} \nu_{i} .
$$

Whence $\lambda_{K}=\lambda_{K} \star \lambda$ follows. I.e., $\operatorname{supp} \lambda \subseteq H_{r}\left(\lambda_{K}\right)$. Again by assumption (iii'), $\lambda_{K} \rightarrow \varepsilon_{e}$, hence $\varepsilon_{e}=\varepsilon_{e} \star \lambda$. I.e., $\lambda=\varepsilon_{e}$, and $\mathcal{L}=\left\{\varepsilon_{e}\right\}$. According to [10], 2.2.16, (i) follows.

Assume (iii), and assume further that $\mathbb{G}$ is aperiodic. As afore mentioned, $\left\{\lambda_{k, n}\right\}$ is relatively compact. Thus, according to [10], 2.2.19, $(i)$ follows.

Assume (iii), and in addition, $H_{r}(\mu)=\{e\}$. As before, for $\lambda \in \mathcal{L}$ we obtain $\mu=\mu \star \lambda$. Again $\operatorname{supp}(\lambda) \subseteq H_{r}(\mu)$ and thus, by assumption, $\lambda=\varepsilon_{e}$. Again, according to [10], 2.2.16, $(i)$ follows.

Note that $H_{r}(\mu)=\{e\} \Rightarrow H_{r}\left(\lambda_{k}\right)=\{e\}$ and, if the measures $\nu_{i}$ belong to a commutative sub-semigroup $\mathcal{S}$ of $\mathcal{M}^{1}(\mathbb{G}), H_{r}\left(\lambda_{k, n}\right)=\{e\}$.

For special products considered in the sequel, $\mu=\underset{i \geq 0}{\star} \tau^{i}(\nu)$, the condition $H_{r}(\mu)=\{e\}$ is superfluous:

Corollary 3.2. Let $\mathbb{G}$ be a contractible locally compact group with contracting $\tau \in \operatorname{Aut}(\mathbb{G})$.

Let $X_{i}, i \geq 0, Y$ be independent random variables with distribution $\nu=X_{i}(P), i \geq 0, \mu:=Y(P)$. Then, with the notations above, $\mathcal{L}=$ $\left\{\varepsilon_{e}\right\}$ and hence

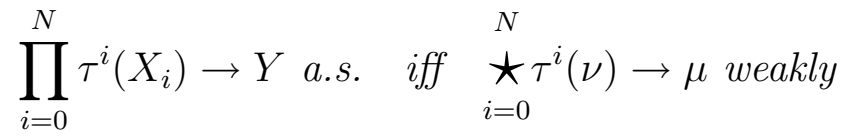

Proof. It suffices to show that condition $(i)$ of Proposition 3.1 is satisfied. If $\mu_{N}:=\underset{j=0}{\star} \tau^{j}(\nu) \stackrel{N \rightarrow \infty}{\rightarrow} \mu$ then for all $K, \lambda_{K, N}:=\underset{j=K+1}{\underset{N}{\star}} \tau^{j}(\nu) \stackrel{N \rightarrow \infty}{\rightarrow}$ $\tau^{K+1}(\mu)=\lambda_{K}$. As $\tau$ is contracting, $\tau^{K+1}(\mu) \rightarrow \varepsilon_{e}$ follows. Let $\lambda \in \mathcal{L}$, let $\lambda_{K_{n}, N_{n}} \rightarrow \lambda$. As $\lambda_{K_{n}, N_{n}} \star \lambda_{N_{n}} \rightarrow \varepsilon_{e}$, it follows $\lambda_{K_{n}, N_{n}} \rightarrow \varepsilon_{e}$ by the shift compactness theorem ([22]), III, theorem 2.1, 2.2, [10], theorem 1.2.21. Hence $\left(i i^{\prime}\right)$ and thus (i) (in Proposition 3.1) follow.

Remark 3.3. We define ad hoc: A infinite convolution product $\mu=$ $\underset{j \geq 0}{\star} \nu_{j}$ converges strongly if all rest products $\lambda_{k}:=\underset{j>k}{\star} \nu_{j}$ are convergent $(k \geq-1)$ and in addition $\lambda_{k} \stackrel{k \rightarrow \infty}{\rightarrow} \varepsilon_{e}$. I.e., if condition $\left(i i i^{\prime}\right)$ in Proposition 3.1 is satisfied. A product of convolution powers $\mu=\underset{j \geq 0}{\star} \nu_{j}^{k(j)}$ converges strongly if it converges strongly in lexicographical order.

a) As shown in Proposition $3.1\left(^{\prime}(i) \Leftrightarrow\left(i i i^{\prime}\right)^{\prime}\right)$, strong convergence implies that the the equivalence theorem holds.

b) Moreover, we define ad hoc for a fixed group norm $|\cdot|$ : If $\left(X_{j}\right)$ are independent $\mathbb{G}$-valued random variables, with distributions $X_{i}(P)=\nu_{i}$ then $\prod X_{j}$ is a.s. absolutely convergent if $\sum\left|X_{i}\right|<\infty$ a.s. Analogously we define for independent arrays $\left(X_{i, j}\right)$ with distributions $X_{i, j}(P)=\nu_{j}$ 
that the iterated products $\prod_{j \geq 0} \prod_{i=1}^{k(j)} X_{i, j}$ converge a.s. absolutely if $\sum_{j} \sum_{i=1}^{k(j)}\left|X_{i, j}\right|<\infty$ a.s.

A.s. absolute convergence implies strong convergence. In fact, a.s. absolute convergence implies a.s. convergence of all partial products and re-arranged products of the above products, and hence weak convergence of the convolution products $\underset{j \geq 0}{\star} \nu_{j}$ resp. $\underset{j \geq 0}{\star} \nu_{j}^{k(j)}$. Moreover, $\sum_{j>K} \sum_{i=0}^{k(j)}\left|X_{j}\right| \rightarrow 0$ a.s. implies that the rest products $\underset{j>K}{\underset{j}{\star}} \nu_{j}^{k(j)}$ converge to $\varepsilon_{e}(K \rightarrow \infty)$.

As well known, on vector spaces and also on homogeneous groups, convolution products $\underset{k+1}{\star} \tau^{j}(\nu)$ converge iff logarithmic moments exist. As in the case of homogeneous groups (cf. [6], [7]) we define logarithmic moments in terms of group norms. (For vector spaces see e.g., [11].)

Definition 3.4. Let $\mathbb{G}$ be a locally compact group with a group norm $|\cdot|$. Let $(\Omega, \Sigma, P)$ be a probability space. Let $X$ be a $\mathbb{G}$-valued random variable with distribution $X(P)=\nu . X$ resp. $\nu$ possesses finite logarithmic moments of order $n$, symbolically $\nu \in \mathcal{P}_{\log _{+}^{n}}$, if $\mathbb{E}\left(\left(\log _{+}|X|\right)^{n}\right)=$ $\int_{\mathbb{G}}\left(\log _{+}|x|\right)^{n} \mathrm{~d} \nu(x)<\infty$, equivalently, if $\int\left(\log _{+}(1+|x|)\right)^{n} \mathrm{~d} \nu(x)<\infty$.

Obviously, we have: Let $f: \Omega \rightarrow \mathbb{R}_{+}$be measurable, $\psi \in C^{1}(\mathbb{R})$ strictly increasing with integrable $\psi^{\prime}$. Then

$$
\int_{\Omega} \psi \circ f \mathrm{~d} P=\int_{\mathbb{R}_{+}} P\{f>y\} \psi^{\prime}(y) \mathrm{d} y
$$

Applying this observation to the functions $t \mapsto \log _{+}(t)$ resp. $t \mapsto$ $\log (1+t)$ we obtain

Lemma 3.5. $\nu \in \mathcal{P}_{\log _{+}^{n+1}}<\infty$ iff one of the following equivalent conditions is satified:

$$
\begin{aligned}
& \mathbb{E}\left(\log (1+|X|)^{n+1}\right)<\infty \Leftrightarrow \int_{0}^{\infty} P\left(|X|>\mathrm{e}^{y}-1\right) y^{n} \mathrm{~d} y<\infty \\
\Leftrightarrow & \int_{0}^{\infty} \nu\left\{|\cdot|>\mathrm{e}^{y}-1\right\} y^{n} \mathrm{~d} y<\infty \Leftrightarrow \mathbb{E}\left(\log _{+}(|X|)^{n+1}\right)<\infty \\
\Leftrightarrow & \int_{1}^{\infty} P\left(|X|>\mathrm{e}^{y}\right) y^{n} \mathrm{~d} y<\infty \Leftrightarrow \int_{1}^{\infty} \nu\left\{|\cdot|>\mathrm{e}^{y}\right\} y^{n} \mathrm{~d} y<\infty
\end{aligned}
$$

Easy calculations show

Lemma 3.6. Let $X$ be a $\mathbb{G}$-valued random variable and $|\cdot|$ a group norm. Then,

$$
\int_{1}^{\infty} P\left\{\log _{+}|X|>y\right\} y^{n} \mathrm{~d} y<\infty \Rightarrow \sum_{1}^{\infty} P\left\{\log _{+}|X|>j \beta\right\} j^{n}<\infty
$$

for all $\beta>0$. And if for some $\beta>0$

$$
\sum_{1}^{\infty} P\left\{\log _{+}|X|>j \beta\right\} j^{n}<\infty \text { then } \int_{1}^{\infty} P\left\{\log _{+}|X|>y\right\} y^{n} \mathrm{~d} y<\infty
$$


【 Note that $f_{1}: y \mapsto P\left(\log _{+}|X|>y\right) \searrow$ and $f_{2}:[1, \infty) \ni y \mapsto$ $y^{n} \nearrow$. This yields upper and lower estimates for the Riemann sums approximating $\int_{1}^{\infty} f_{1} \cdot f_{2} \mathrm{~d} y$. ]

Proposition 3.7. Let $\mathbb{G}$ be a contractible locally compact group with contracting $\tau \in \operatorname{Aut}(\mathbb{G})$ and group norm $|\cdot|$ satisfying the growth condition (2.1). Let, for fixed $n \in \mathbb{N}, k(j), j \geq 1$, be a sequence in $\mathbb{N}$ such that $k(j) / j^{n}$ is bounded. (E.g., $k(j):=\left(\begin{array}{c}n+j \\ n\end{array}\right)$, as throughout in the sequel). Then we have:

$$
\nu \in \mathcal{P}_{\log _{+}^{n+1}} \Rightarrow \mu:=\underset{j \geq 0}{\star} \tau^{j}\left(\nu^{k(j)}\right) \quad \text { is weakly convergent }
$$

Moreover, let $\left(X_{i ., j}\right): 1 \leq i \leq k(j), j \geq 0,\left(X_{i}\right): i \geq 0$, be iid arrays with distribution $X_{i, j}(P)=\nu, X_{i}(P)=\nu$. Then

$$
\sum_{j \geq 0} \sum_{i=1}^{k(j)}\left|\tau^{j}\left(X_{i, j}\right)\right|<\infty \text { a.s. and } \sum_{j \geq 0} k(j)\left|\tau^{j}\left(X_{j}\right)\right|<\infty \text { a.s. }
$$

In particular, $\prod_{j \geq 0} \prod_{i=1}^{k(j)} \tau^{j}\left(X_{i, j}\right)$ is a.s. convergent and re-arranged products and partial products are convergent.

(For real vector spaces see e.g., [11], Lemma 3.6.5, for $n=0$, or [16], for $n \geq 1$.)

Proof. 1). Assume first the growth condition (2.1) holds true with $m_{1}=1$.

Let $X, X_{i}, X_{i, j}$ be iid random variables with distribution $\nu$. Assume $\nu \in \mathcal{P}_{\log _{+}^{n+1}}$ for some (fixed) $n \geq 0$. As afore mentioned, in Lemma 3.5, we have $\int_{0}^{\infty} P\left(\log _{+}|X|>y\right) y^{n} \mathrm{~d} y<\infty$. And, according to Lemma 3.6, for any $\alpha>0$ this is equivalent with

$$
\begin{aligned}
& \sum_{j=0}^{\infty} j^{n} P\left(\log _{+}|X|>j \alpha\right)<\infty \Leftrightarrow \sum_{j=0}^{\infty} j^{n} P\left(|X|>\left(\mathrm{e}^{\alpha}\right)^{j}\right)<\infty \\
\Leftrightarrow & I:=\sum_{j=0}^{\infty} \sum_{i=1}^{j^{n}} P\left(\left|X_{i, j}\right|>\left(\mathrm{e}^{\alpha}\right)^{j}\right)<\infty
\end{aligned}
$$

The growth condition (2.1) yields $r^{n} \cdot|x| \leq\left|\tau^{n} x\right| \leq R^{n} \cdot|x| \forall x$, and hence for all $\delta>0$

$$
\left\{\left|\tau^{n} x\right| \geq r^{n} \delta\right\} \supseteq\{|x| \geq \delta\} \supseteq\left\{\left|\tau^{n} x\right| \geq R^{n} \delta\right\}
$$

Inserting $\delta=\mathrm{e}^{\alpha j}$ yields

$$
\begin{aligned}
& P\left(\left|X_{i, j}\right| \geq\left(\mathrm{e}^{\alpha}\right)^{j}\right) \leq P\left(\left|\tau^{j}\left(X_{i, j}\right)\right| \geq\left(r \cdot \mathrm{e}^{\alpha}\right)^{j}\right) \\
& P\left(\left|X_{i, j}\right| \geq\left(\mathrm{e}^{\alpha}\right)^{j}\right) \geq P\left(\left|\tau^{j}\left(X_{i, j}\right)\right| \geq\left(R \cdot \mathrm{e}^{\alpha}\right)^{j}\right)
\end{aligned}
$$

and thus

$$
\begin{aligned}
& \sum_{j=0}^{\infty} \sum_{i=1}^{j^{n}} P\left(\left|\tau^{j}\left(X_{i, j}\right)\right| \geq\left(R \mathrm{e}^{\alpha}\right)^{j}\right) \leq I \\
& \leq \sum_{j=0}^{\infty} \sum_{i=1}^{j^{n}} P\left(\left|\tau^{j}\left(X_{i, j}\right)\right| \geq\left(r \mathrm{e}^{\alpha}\right)^{j}\right)
\end{aligned}
$$


Hence, with $\delta_{\alpha}:=R \cdot \mathrm{e}^{\alpha}$ we obtain $\sum_{j=0}^{\infty} \sum_{i=1}^{j^{n}} P\left(\left|\tau^{j}\left(X_{i, j}\right)\right| \geq \delta_{\alpha}^{j}\right)<\infty$.

Put, for $1 \leq i \leq j^{n}, j \geq 1$, and fixed $\alpha>0, A_{i, j}:=\left\{\left|\tau^{j}\left(X_{i, j}\right)\right| \geq \delta_{\alpha}^{j}\right\}$. Then, by the Lemma of Borel-Cantelli, $P\left(A^{*}\right)=0$, where $A^{*}:=$ $\operatorname{Limsup} A_{k . \ell}$ (in lexicographical order). I.e., considering $\complement A^{*}$, we obtain: For $P$-almost all $\omega$ there exists $j(\omega)$ such that for all $i \leq j^{n}$, $j \geq j(\omega)$ we have: $\omega \in \complement A_{i, j}$, i.e., $\left|\tau^{j}\left(X_{i, j}(\omega)\right)\right|<\delta_{\alpha}^{j}$.

Hence, for small $\alpha$, such that $\delta_{\alpha}=\operatorname{Re}^{\alpha}<1$,

$$
\begin{aligned}
& \sum_{j=0}^{\infty} \sum_{i=1}^{j^{n}}\left|\tau^{j}\left(X_{i, j}\right)\right| \\
= & \sum_{j=0}^{j(\omega)} \sum_{i=1}^{j^{n}}\left|\tau^{j}\left(X_{i, j}\right)\right|+\sum_{j>j(\omega)} \sum_{i=1}^{j^{n}}\left|\tau^{j}\left(X_{i, j}\right)\right| \\
\leq & c_{1}(\omega)+\sum_{j \geq 0} \sum_{i=1}^{j^{n}} \delta_{\alpha}^{j}=c_{1}(\omega)+\sum_{j \geq 0} j^{n} \delta_{\alpha}^{j}<\infty P-\text { a.s. }
\end{aligned}
$$

with some constant $c_{1}(\omega)$.

Analogously, $\sum_{j \geq 0} j^{n}\left|\tau^{j}\left(X_{i, j}\right)\right| \leq c_{2}(\omega)+\sum_{j \geq 0} j^{n} \delta_{\alpha}^{j}<\infty P$-a.s.

2) In the case $m_{1}>1$ the proof runs analogously, the above estimates hold true only for $j \geq m_{1}$. Hence in the estimates for the sums $\sum_{j=0}^{\infty} \sum_{i=1}^{j^{n}}\left|\tau^{j}\left(X_{i, j}\right)\right|$ etc. additional additive constants (depending on $\omega$ ) occur.

A.s. absolute convergence yields that $\prod_{j \geq 0} \prod_{i=1}^{j^{n}} \tau^{j}\left(X_{i, j}\right)$ as well as all partial products and re-arranged products converge a.s. (Remark 3.3). In particular, weak convergence of $\underset{j \geq 0}{\star} \tau^{j}\left(\nu^{k(j)}\right)$ follows.

By assumption, $k(j) \leq C \cdot j^{n}$, whence $\sum_{j=0}^{\infty} \sum_{i=1}^{k(j)}\left|\tau^{j}\left(X_{i, j}\right)\right|<\infty P$-a.s. follows.

Converse results: For homogeneous groups we obtain as in the case of vector spaces (in analogy to e.g., [16], [17]):

Proposition 3.8. Let $\mathbb{G}$ be a homogeneous group with contracting $\tau \in$ $\operatorname{Aut}(\mathbb{G})$ and corresponding group norm $|\cdot|$. Let again $k(j) \approx j^{n}$, e.g., $k(j)=\left(\begin{array}{c}n+j \\ n\end{array}\right)$. Then we have

$$
\underset{j \geq 0}{\star} \tau^{j}\left(\nu^{k(j)}\right) \text { converges weakly } \Rightarrow \nu \in \mathcal{P}_{\text {log }_{+}^{n+1}}
$$

Proof. $\mathbb{G}$ is a homogeneous group, hence aperiodic. According to the equivalence theorem Proposition 3.1, weak convergence of the products $\underset{j \geq 0}{\star} \tau^{j}\left(\nu^{k(j)}\right)$ implies a.s. convergence of $\prod_{j \geq 0} \prod_{i=1}^{k(j)} \tau^{j}\left(X_{i, j}\right)$ for iid random 
variables with distribution $X_{i, j}(P)=\nu$. Hence we obtain:

$P\left(\bigcap_{n \geq 0}\left(\bigcup_{j \geq n, i \leq k(j)}\left\{\left|\tau^{j} X_{i, j}\right|>\delta\right\}\right)\right)=0$ for all $\delta>0$.

According to the Lemma of Borel-Cantelli it follows for all $\delta>0$ :

$$
I:=\sum_{j=0}^{\infty} \sum_{i=0}^{j^{n}} P\left(\left|\tau^{j} X_{i, j}\right|>\delta\right)=\sum_{j \geq 0} j^{n} P\left(\left|\tau^{j} X_{i, j}\right|>\delta\right)<\infty
$$

since the variables $X_{i, j}$ are identically distributed and $k(j) \approx j^{n}$. The growth condition $(2.1$ ) yields (for all $i$ )

$$
\left\{\left|\tau^{j} X_{i, j}\right|>\delta\right\}=\left\{\left|\tau^{j} X_{i, j}\right|>r^{j} r^{-j} \delta\right\} \supseteq\left\{\left|X_{i, j}\right|>r^{-j} \delta\right\}
$$

We have $r^{-1}>1$. Choose $\delta>1$ and put $\gamma:=r^{-1} \delta, \gamma_{j}:=\delta^{1 / j} r^{-1} \leq \gamma$. Since $P\left(|X|>\gamma_{j}^{j}\right) \geq P\left(|X|>\gamma^{j}\right), j \in \mathbb{Z}_{+}$

$$
\infty>I \geq \sum_{j \geq 0} j^{n} P\left(\left|X_{i, j}\right|>\delta r^{-j}=\gamma_{j}^{j}\right)=\sum_{j \geq 0} j^{n} P\left(\left|X_{i, j}\right|>\gamma^{j}\right)
$$

and hence (with $\beta=\log \gamma \geq 0$ ):

$$
\infty>I \geq \sum_{j \geq 0} j^{n} P\left(\log \left|X_{i, j}\right|>\beta j\right)
$$

Thus, by Lemma 3.6, $\int_{1}^{\infty} P\left(\log _{+}|X|>y\right) y^{n} \mathrm{~d} y<\infty$, whence $\nu \in$ $\mathcal{P}_{\log _{+}^{n+1}}$.

The proof of Proposition 3.8 shows that the following more general result holds true:

Proposition 3.9. Let $\mathbb{G}$ be a contractible locally compact group with contracting $\tau \in$ Aut $(\mathbb{G})$, let $|\cdot|$ be a group norm adapted to $\tau$ and satisfying (2.1). Let $k(j) \approx\left(\begin{array}{c}n+j \\ n\end{array}\right)$. Let $\left(X_{i, j}\right)_{i, j \geq 0}$ be independent random variables with distribution $\nu$. Assume that convergence of the convolution product $\mu=\underset{j=0}{\star} \tau^{k(j)}(\nu)$ implies a.e. convergence of $\prod_{j=0}^{\infty} \prod_{i=0}^{k(j)} X_{i, j}$. Then we have $\mu \in \stackrel{j}{\mathcal{P}}_{\log _{+}^{n+1}}$.

The condition is satisfied in the case $n=0$ (Corollary 3.2), or if the product converges strongly (in the sense of Remark 3.3) or if $H_{r}(\mu)=$ $\{e\}$, (or if $\mathbb{G}$ is a homogeneous group, as in Proposition 3.8).

Proof. Analogous to the proof of Proposition 3.8: There $\mathbb{G}$ was supposed to be a homogeneous group just in order to apply the equivalence theorem, Proposition $3.1^{\prime}($ iii $) \Rightarrow(i)^{\prime}$ '. Note that for $n=0$, weak and a.e. convergence are equivalent by Corollary 3.2. Analogously, if strong convergence or $H_{r}(\mu)=\{e\}$ or, more generally, $\mathcal{L}=\left\{\varepsilon_{e}\right\}$, is assumed, a.s. convergence follows by Remark 3.3 (resp. Proposition 3.1).

Remarks 3.10. a) We have shown by the preceding Propositions 3.7 - 3.8 that for homogeneous groups, $\forall n \in \mathbb{Z}_{+}$, for any group norm - not only homogeneous norm - satisfying a growth condition (2.1) the existence of logarithmic moments $\nu \in \mathcal{P}_{\log _{+}^{n+1}}$ characterizes weak convergence $\underset{j \geq 0}{\star} \tau^{j}\left(\nu^{\left(\begin{array}{c}n+j \\ n\end{array}\right)}\right)$. This generalizes e.g., [6], Proposition 3.4, 
Theorem 3.5, resp. [7], Proposition 2.14.24, for the case $n \geq 0$. In particular this proves that $\mathcal{P}_{\log _{+}^{n+1}}$ does not depend on the particular chosen group norm.

Analogously, for $n=0$, Proposition 3.9, generalizes the above mentioned results in [6], [7], to general contractible locally compact groups. Again this implies that $\mathcal{P}_{\log _{+}}$does not depend on the particular chosen group norm.

b) A.s. absolute convergence, $\sum_{j \geq 0} \sum_{i=1}^{\left(\begin{array}{c}n+j \\ n\end{array}\right)}\left|\tau^{j} X_{i, j}\right|<\infty$ a.s., implies weak convergence of re-arranged products and of partial products of $\underset{j}{\star} \tau^{j}\left(\nu^{\left(\begin{array}{c}n+j \\ n\end{array}\right)}\right)$. (Cf. Propositions 3.7, (3.1 )) We obtain therefore:

b1) Let $\mathbb{G}$ be contractible with contracting $\tau \in \operatorname{Aut}(\mathbb{G})$. Assume that $\nu_{(k)}:=\underset{j \geq 0}{\star} \tau^{j}\left(\nu^{\left(\begin{array}{c}k+j \\ k\end{array}\right)}\right)$ is convergent for all $k \leq n-1$. Let $\left(Z_{j}^{(k)}\right)$ be corresponding random variables with distributions $Z_{j}^{(k)}(P)=\nu_{(k)}$. Then we have by Corollary 3.2, resp. Proposition 3.8: $\prod_{j \geq 0} \tau^{j}\left(Z_{j}^{(k)}\right)$ converges a.s. absolutely for all $k \leq n-1$, i.e., $\sum_{j \geq 0}\left|\tau^{j}\left(Z_{j}^{(k)}\right)\right|<\infty$ a.s.

b2) Furthermore, let $\left(Z^{(n)}\right),\left(Z_{j}^{(n-1)}\right),\left(Z_{i, j}^{(n-2)}\right), \ldots$ be sequences of independent random variables with distributions $\nu_{(n)}, \nu_{(n-1)}, \ldots$. Then:

$$
\begin{aligned}
Z^{(n)} & =\prod_{j \geq 0} \tau^{j}\left(Z_{j}^{(n-1)}\right)=\prod_{j \geq 0} \tau^{j} \prod_{i \geq 0} \tau^{i}\left(Z_{j, i}^{(n-2)}\right) \\
& =\cdots=\prod_{j_{n} \geq 0} \tau^{j_{n}}\left(\prod_{j_{n-1} \geq 0} \tau^{j_{n-1}}\left(\cdots\left(\prod_{j_{0} \geq 0} \tau^{j_{0}}\left(Z_{j_{n}, \cdots, j_{0}}^{(0)}\right) \cdots\right)\right)\right) \\
& =\prod_{j_{n} \geq 0}\left(\prod_{j_{n-1} \geq 0}\left(\cdots\left(\prod_{j_{0} \geq 0} \tau^{\sum_{0}^{n} j_{i}}\left(Z_{j_{n}, \cdots, j_{0}}^{(0)}\right) \cdots\right)\right)\right)
\end{aligned}
$$

b3) If in b2), $\mathbb{G}$ is a homogeneous group or if one of the conditions in Proposition 3.9 is satisfied, then $\nu \in \mathcal{P}_{\log _{+}^{n+1}}$ and (Proposition 3.7) a.s. convergence of the random variables $\sum_{j_{n}, \ldots, j_{0}}\left|\tau^{\sum j_{i}}\left(X_{j_{n}, \ldots, j_{0}}\right)\right|$ follows.

\section{Multiple $\tau$-Decomposability of Probabilities}

Let in this section, $\mathbb{G}$ be a contractible locally compact group with contracting $\tau \in \operatorname{Aut}(\mathbb{G})$. Let furthermore, $|\cdot|$ be a group norm adapted to $\tau$ satisfying the growth condition (2.1).

Definition 4.1. a) $\mu \in \mathcal{M}^{1}(\mathbb{G})$ is $\tau$-decomposable - in symbols, $\mu \in$ $L_{0}(\tau)$ - with cofactor $\nu \in \mathcal{M}^{1}(\mathbb{G})$ if $\mu=\nu \star \tau(\mu)$.

In terms of random variables: A random variable $X$ with distribution $\mu$ is $\tau$-decomposable if there exists a random variable $Y$, such that $(X, Y)$ are independent and $X \stackrel{d}{=} Y \cdot \tau(X)$ (equality of distributions). 
b) Put $L_{-1}(\tau):=\mathcal{M}^{1}(\mathbb{G})$. $\mu \in \mathcal{M}^{1}(\mathbb{G})$ is n-times $\tau$-decomposable - in symbols, $\mu \in L_{n}(\tau)$ - if, $\mu$ is $\tau$-decomposable, $\mu=\nu_{(n)} \star \tau(\mu)$, with cofactor $\nu_{(n)} \in L_{n-1}(\tau)$.

Therefore, $\mu=\nu_{(n)} \star \tau(\mu), \quad \nu_{(n)}=\nu_{(n-1)} \star \tau\left(\nu_{(n)}\right), \quad \cdots, \nu_{(1)}=$ $\nu \star \tau\left(\nu_{(1)}\right)$. Hence $\mu=\nu \star \tau\left(\nu_{(1)}\right) \star \cdots \star \tau\left(\nu_{(n)}\right) \star \tau(\mu)$

If the contraction condition is not supposed, $\mu$ is usually called strongly $\tau$-decomposable if in addition $\tau^{k}(\nu) \rightarrow \varepsilon_{e}$. Here, as we always restrict to contracting $\tau$, 'strongly' is superfluous.

Proposition 4.2. a) $\mu$ is $\tau$-decomposable with cofactor $\nu$ iff

$$
\mu=\underset{j=0}{\star} \tau^{j}(\nu):=\lim _{N \rightarrow \infty} \nu \star \cdots \tau^{N}(\nu) .
$$

b) $\mu$ is n-times $\tau$-decomposable iff

$$
\mu=\underset{j_{n} \geq 0}{\star} \underset{j_{n-1} \geq 0}{\star}\left(\cdots \underset{j_{0} \geq 0}{\star} \underset{\left.\tau^{\sum_{0}^{n} j_{i}}(\nu) \cdots\right)}{\star}\right. \text {. }
$$

Proof. a) ' $\Rightarrow$ Obvious, since for all $N$ we have $\underset{0}{\star} \star \tau^{j}(\nu) \star \tau^{N+1}(\mu)=$ $\mu$ and, since $\tau$ is contracting, thus $\tau^{N+1}(\mu) \rightarrow \varepsilon_{e}$. The assertion follows by the shift compactness theorem, [22], [10].

$$
' \Leftarrow{ }^{\prime} \text { Assume } \mu=\underset{0}{\star} \tau^{k}(\nu) \text {. Hence } \mu=\nu \star \underset{1}{\star} \underset{1}{\infty}(\nu)=\nu \star \tau(\mu) .
$$

(For homogeneous groups see e.g., [6], [7].)

b) follows inserting in a) successively $\nu_{(i)}=\underset{0}{\star} \tau^{k}\left(\nu_{(i-1)}\right), \nu_{(0)}=\nu$.

Hence according to Propositions 3.7, 3.8, 3.9 we obtain immediately:

Proposition 4.3. $\nu \in \mathcal{M}^{1}(\mathbb{G})$ is the cofactor of a $\tau$-decomposable law iff $\nu \in \mathcal{P}_{\log _{+}}$.

We mention two results concerning the structure of $\tau$-decomposable laws. The proofs are left to the reader.

Proposition 4.4. a) The set of $\tau$-decomposable laws is weakly closed.

b) If $\mu_{i}$ are $\tau$-decomposable with cofactors $\nu_{i}$ and if $\mu_{i}, \nu_{i}, i=1,2$, belong to a commutative sub-semigroup $\mathcal{S} \subseteq \mathcal{M}^{1}(\mathbb{G})$, with $\tau(\mathcal{S}) \subseteq \mathcal{S}$, then $\mu_{1} \star \mu_{2}$ is $\tau$-decomposable with cofactor $\nu_{1} \star \nu_{2}$.

The following Lemma will be essential in the sequel. Since compact subgroups are not excluded, a careful discussion of convergence and re-arranging of iterated convolution products seems to be necessary.

Lemma 4.5. Let, as before, $\mathbb{G}$ be contractible with contracting $\tau \in$ Aut $(\mathbb{G})$, let $\mathcal{S} \subseteq \mathcal{M}^{1}(\mathbb{G})$ be a commutative sub-semigroup with $\tau \mathcal{S} \subseteq \mathcal{S}$. Let $\mu, \nu \in \mathcal{S}$. Let $n \in \mathbb{N}$. If $n>1$ we assume in addition $H_{r}(\mu)=\{e\}$. Then the following assertions are equivalent:

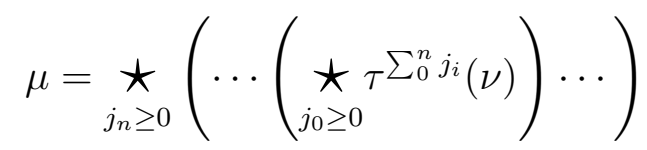




$$
\mu=\underset{j \geq 0}{\star} \tau^{j}\left(\nu^{k_{n}(j)}\right) \quad \text { with } \quad k_{n}(j)=\left(\begin{array}{c}
n+j \\
n
\end{array}\right)
$$

Proof. $\quad '(4.1) \Rightarrow(4.2)^{\prime}$. We proceed by induction on $n$. Let $n=1$. Then $\mu=\underset{j \geq 0}{\star} \tau^{j}\left(\underset{i \geq 0}{\star} \tau^{i}(\nu)\right)=\underset{j \geq 0}{\star} \tau^{j}\left(\nu_{(1)}\right)$ and $\nu_{(1)}:=\underset{i \geq 0}{\star} \tau^{i}(\nu)$ are both $\tau$-decomposable and $\nu, \nu_{(1)} \in \mathcal{P}_{\log _{+}}$(Propositions 3.7, 3.9). For all $L, M \in \mathbb{N}$ we have:

$$
\begin{aligned}
& \mu=\underset{i=0}{\star} \tau^{i}\left(\nu_{(1)}\right) \star \tau^{L+1}(\mu) \\
& \nu_{(1)}=\underset{i=0}{\star} \underset{\tau^{i}}{M}(\nu) \star \tau^{M+1}\left(\nu_{(1)}\right)
\end{aligned}
$$

Hence for all $L \in \mathbb{N}$, inserting successively (4.4) in (4.3) with $M=L-\ell$ we obtain (in view of $\nu \in \mathcal{S}$ ):

$$
\begin{aligned}
& \mu=\underset{\ell=0}{\star} \tau^{\ell}\left(\prod_{j=0}^{L-\ell} \tau^{j}(\nu) \star \tau^{L-\ell+1}\left(\nu_{(1)}\right)\right) \star \tau^{L+1}(\mu) \\
&= \lambda_{L}^{(1)} \star \tau^{L+1}\left(\nu_{(1)}^{L+1}\right) \star \tau^{L+1}(\mu) \\
& \text { where } \lambda_{L}^{(1)}=\underset{\ell=0}{\star} \tau^{\ell}\left({ }_{j=0}^{L-\ell} \tau^{j}(\nu)\right)=\underset{i=0}{\star}(\underbrace{i}_{j=0} \tau^{i}(\nu))=\underset{i=0}{\star} \tau^{i}\left(\nu^{i+1}\right)
\end{aligned}
$$

and $\tau^{L+1}(\mu) \rightarrow \varepsilon_{e}$. We have to show that $\lambda_{L}^{(1)} \rightarrow \mu$, equivalently, $\tau^{L+1}\left(\nu_{(1)}^{L+1}\right) \rightarrow \varepsilon_{e}$. Hence $\mu=\underset{0}{\star} \underset{\sim}{\infty} \tau^{i}\left(\nu^{i+1}\right)$, i.e., ( 4.2$)$ for $n=1$.

Since $\nu_{(1)} \in \mathcal{P}_{\log _{+}}$, for a iid sequence $\left(Y_{j}\right)$ of random variables with distribution $\nu_{(1)}$ we obtain $\sum\left|\tau^{j}\left(Y_{j}\right)\right|<\infty$ a.s. (Proposition $3.7(3.1)$ ). $\tau$ satisfies the growth condition (2.1), $\left\|\tau^{k}\right\| \leq R^{k}, k \geq m_{1}$, for $0<R<$ 1. Let $C:=\max \left\|\tau^{k}\right\|(\geq 1)$. Let $L+1=N_{1}+N_{2}, N_{i}>m_{1}$. Then we obtain:

$$
\begin{aligned}
& \left|\tau^{L+1}\left(\prod_{0}^{L+1} Y_{j}\right)\right| \leq \sum_{0}^{N_{1}}\left|\tau^{L+1}\left(Y_{j}\right)\right|+\sum_{N_{1}+1}^{L+1}\left|\tau^{L+1}\left(Y_{j}\right)\right| \\
\leq & R^{N_{2}} \sum_{0}^{N_{1}}\left|\tau^{N_{1}}\left(Y_{j}\right)\right|+\sum_{N_{1}+1}^{L+1}\left|\tau^{L+1}\left(Y_{j}\right)\right| \\
\leq & R^{N_{2}} \cdot C \cdot\left(\sum_{0}^{\infty}\left|\tau^{j}\left(Y_{j}\right)\right|\right)+C \cdot\left(\sum_{N_{1}+1}^{\infty}\left|\tau^{j}\left(Y_{j}\right)\right|\right) \stackrel{\left(N_{1}, N_{2}\right) \rightarrow \infty}{\longrightarrow} 0 \text { a.s. }
\end{aligned}
$$

In fact, we have $\left|\tau^{N_{1}}\left(Y_{j}\right)\right| \leq \| \tau^{N_{1}-j}||\left|\tau^{j}\left(Y_{j}\right)\right| \leq C \cdot\left|\tau^{j}\left(Y_{j}\right)\right|$ for $j \leq N_{1}$, and $\left|\tau^{L+1}\left(Y_{j}\right)\right| \leq\left\|\tau^{L+1-j}\right\|\left|\tau^{j}\left(Y_{j}\right)\right| \leq C \cdot\left|\tau^{j}\left(Y_{j}\right)\right|$ for $N_{1}<j \leq L+1$.

Let the assertion be proved for $k \leq n$. We assumed $H_{r}(e)=$ $\{e\}$, hence Proposition 3.9 is applicable. Let $\nu_{(0)}=\nu, \ldots, \nu_{(i)}=$ $\underset{k \geq 0}{\star} \tau^{k}\left(\nu_{(i-1)}\right), \nu_{(n+1)}=\mu$. By assumption, $\mu=\underset{j=0}{\underset{j}{\star} \tau^{j}}\left(\nu_{(n)}\right)$ and by 
induction hypothesis, $\nu_{(n)}=\underset{j=0}{\star} \tau^{j}\left(\nu^{k_{n}(j)}\right)$ with $k_{n}(j)=\left(\begin{array}{c}n+j \\ n\end{array}\right)$. Hence $\mu \in \mathcal{P}_{\log _{+}}$and $\nu_{(n)} \in \mathcal{P}_{\log _{+}{ }^{n+1}}$.

Hence for all arrays $\left(X_{i, j}\right)$ of iid random variables with distribution $\nu$ we have a.s. absolute convergence, $\sum_{j>0} \sum_{i=0}^{k_{n}(j)}\left|\tau^{j}\left(X_{i, j}\right)\right|<\infty$ a.s. Furthermore, for all $L>0$ we have

$$
\mu=\underset{\ell=0}{\star} \tau^{\ell}\left(\nu_{(1)}\right) \star \tau^{L+1}(\mu)=: \mu_{L} \star \tau^{L+1}(\mu)
$$

and (since all measures belong to $\mathcal{S}$ )

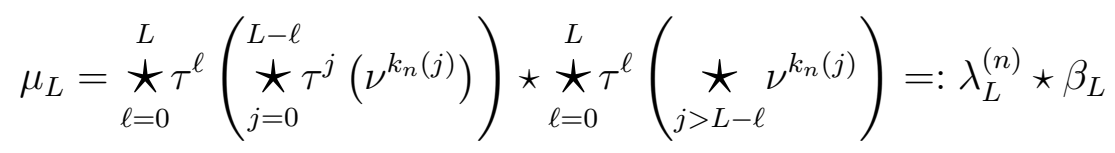

As in the case $n=1$ we observe

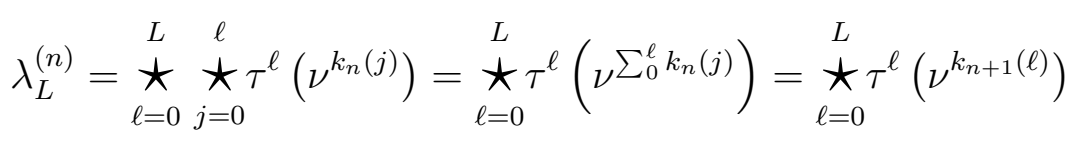

Hence we have to show $\beta_{L} \rightarrow \varepsilon_{e}$. Then $\lambda_{L}^{(n)} \rightarrow \mu$, hence (4.2) is proved.

Let $Z_{L}$ be distributed according to $\beta_{L}$. Since $\nu \in \mathcal{S}$, we may assume $Z_{L}=\prod_{\ell=0}^{L} \tau^{\ell}\left(\prod_{j=L-\ell+1}^{\infty} \tau^{j}\left(\prod_{i=0}^{k_{n}(j)} X_{i, j}\right)\right)$.

We have $\left|Z_{L}\right| \leq \sum_{\ell=0}^{L} C R^{\ell}\left(\sum_{j>L-\ell} \sum_{i=0}^{k_{n}(j)}\left|\tau^{j}\left(X_{i, j}\right)\right|\right)=: U_{L}$. Put for $K \in \mathbb{Z}_{+}, f_{K}:=\sum_{j \geq K} \sum_{i=0}^{k_{n}(j)}\left|\tau^{j}\left(X_{i, j}\right)\right|$. Recall that $f_{K} \searrow 0$ a.s., in particular, $f_{K} \leq f_{0}<\infty$ a.s. Let as in the case $n=1, L=$ $N_{1}+N_{2}$ with $N_{i}>m_{1}$ and decompose $U_{L}=C \cdot \sum_{\ell=0}^{N_{1}} R^{\ell} f_{L-\ell}+C$. $\sum_{\ell=N_{1}+1}^{L} R^{\ell} f_{L-\ell}=: U_{N_{1}}^{L}+V_{N_{1}}^{L}$. In view of $U_{N_{1}}^{L} \leq C \cdot\left(\sum_{\ell \geq 0} R^{\ell}\right) \cdot f_{N_{2}}$ and $V_{N_{1}}^{L} \leq C \cdot\left(\sum_{\ell \geq N_{1}+1} R^{\ell}\right) \cdot f_{0}$ we obtain $U_{L} \stackrel{\left(N_{1}, N_{2}\right) \rightarrow \infty}{\longrightarrow} 0$ a.s., hence $Z_{L} \rightarrow e$ a.s., and thus $\beta_{L} \rightarrow \varepsilon_{e}$. The assertion '(4.1) $\Rightarrow(4.2)^{\prime}$ ' is proved. Note furthermore that (4.2) implies $\nu \in \mathcal{P}_{\log _{+}^{n+2}}$ (Proposition 3.9).

$$
'(4.2) \Rightarrow(4.1) ' \text { Assume } \mu=\underset{j \geq 0}{\underset{j}{*} \tau^{j}}\left(\nu^{k_{n}(j)}\right) \text {. As before, } \nu \in \mathcal{P}_{\log _{+}^{n+1}}
$$

follows, and for all iid arrays $\left(X_{i, j}\right)$ with distribution $\nu$ we have a.s. absolute convergence, $\sum_{j \geq 0} \sum_{i=0}^{k_{n}(j)}\left|\tau^{j}\left(X_{i, j}\right)\right|<\infty$ a.s..

Let $\left(Y_{j_{0}, \ldots, j_{n}}\right)$ be iid random variables with distribution $\nu$. Let, for fixed $j,\left\{X_{i, j}: 1 \leq i \leq k_{n}(j)\right\}$ denote the re-arranged random variables $\left\{Y_{j_{0}, \ldots, j_{n}}: \sum_{0}^{n} j_{i}=j\right\}$. Then $\sum_{j_{n}} \cdots \sum_{j_{0}}\left|\tau^{\sum_{0}^{n} j_{i}}\left(Y_{j_{0},, j_{n}}\right)\right|=$ $\sum_{j \geq 0} \sum_{i=0}^{k_{n}(j)}\left|\tau^{j}\left(X_{i, j}\right)\right|<\infty$ and $\sum_{\left\{j_{n}, \ldots, j_{0}\right\} \sum_{0}^{n} j_{i}>N}\left|\tau^{\sum_{0}^{n} j_{i}}\left(Y_{j_{0},, j_{n}}\right)\right| \stackrel{N \rightarrow \infty}{\longrightarrow} 0$ a.s .

Hence for all $\left(M_{0}, \ldots, M_{n}\right) \in \mathbb{Z}_{+}^{n+1}$ the random variables $U_{M_{0}, \ldots, M_{n}}:=$ $\prod_{j_{n}=0}^{M_{n}} \cdots \prod_{j_{0}=0}^{M_{0}} \tau^{\sum_{0}^{n} j_{i}}\left(Y_{j_{0}, \ldots j_{n}}\right)$ are distributed according to $\lambda_{M_{0}, \ldots, M_{n}}:=$ $\underset{j_{n}=0}{M_{n}} \cdots \underset{j_{0}=0}{\star} \tau^{\sum_{0}^{n} j_{i}}(\nu)$. Furthermore, with $Z_{M_{0}, \ldots, M_{n}}:=\complement \bigotimes\left\{0, \ldots, M_{i}\right\}$, 
we have $\sum_{\left(j_{0}, \ldots, j_{n}\right) \in Z_{M_{0}, \ldots, M_{n}}}\left|\tau^{\sum_{0}^{n} j_{i}}\left(Y_{j_{0}, \ldots, j_{n}}\right)\right| \rightarrow 0$ a.s. if $\left(M_{0}, \ldots, M_{n}\right) \rightarrow$ $\infty$ (since $\sum_{0}^{n} j_{i}>\max \left\{M_{0}, \ldots M_{n}\right\}$ for $\left.\left(j_{0}, \ldots, j_{n}\right) \in Z_{M_{0}, \ldots, M_{n}}\right)$. Thus $U_{M_{0}, \ldots, M_{n}}$ is a.s. convergent, to some random variable $U$ say, for any sequence $\left(M_{0}, \ldots, M_{n}\right) \rightarrow \infty$. Hence $\lambda_{M_{0}, \ldots, M_{n}} \rightarrow \mu^{\prime}$. The Cauchy property yields that $\mu^{\prime}$, the distribution of $U$, does not depend on the particular sequence $\left(M_{0}, \ldots, M_{n}\right) \rightarrow \infty$. Therefore, $\mu^{\prime}=\star \cdots \star \tau^{\sum_{0}^{n} j_{i}}(\nu)$ follows. I.e., (4.2) holds. But then by the step ' $(4.1) \stackrel{j_{0}}{\Rightarrow}(4.2)^{j_{n}}, \mu^{\prime}=\mu$ follows.

The proof is complete.

Putting things together we obtain the following result which contains Theorem 1.1:

Proposition 4.6. Let $\mathbb{G}$ be contractible with contracting $\tau \in \operatorname{Aut}(\mathbb{G})$. Let $\mathcal{S} \subseteq \mathcal{M}^{1}(\mathbb{G})$ be a commutative sub-semigroup with $\tau \mathcal{S} \subseteq \mathcal{S}$ and assume in the sequel that $\nu$ and all cofactors belong to $\mathcal{S}$. Consider the following conditions:

a) $\mu$ is $n$-times $\tau$-decomposable, $\mu=\nu_{(n)} \star \tau(\mu), \ldots, \nu_{(1)}=\nu \star$ $\tau\left(\nu_{(1)}\right)$.

b1) $\nu \in \mathcal{P}_{\log _{+}^{n+1}}$ b2) $\mu=\underset{k \geq 0}{\star} \tau^{k}\left(\nu^{\left(\begin{array}{c}n+k \\ n\end{array}\right)}\right)$ is convergent

b3) $\nu_{(k)}=\underset{j \geq 0}{\star} \tau^{j}\left(\nu^{\left(\begin{array}{c}k+j \\ k\end{array}\right)}\right)$ is convergent $\forall k \leq n+1, \nu_{(n+1)}=\mu$.

c) For an iid sequence $X_{i, j}$ with distribution $X_{i, j}(P)=\nu$ the products $\prod_{j \geq 0} \prod_{i=1}^{\left(\begin{array}{c}n+j \\ n\end{array}\right)} \tau^{j} X_{i, j}$ converge a.s. absolutely, i.e., $\sum_{j \geq 0} \sum_{i=1}^{\left(\begin{array}{c}n+j \\ n\end{array}\right)}\left|\tau^{j} X_{i, j}\right|<\infty$ a.s.

Then it follows:

$b 1) \Rightarrow b 2)$, b3), and c) (with cofactors $\nu_{(k)}$ as in b3)), moreover, $b 1) \Rightarrow a)$.

And if in addition $H_{r}(\mu)=\{e\}$ then $\left.\left.a\right) \Rightarrow b 2\right)$ and $\left.\left.b 2\right) \Rightarrow b 1\right)$.

The condition $H_{r}(\mu)=\{e\}$ can be replaced by any other condition of Proposition 3.9 providing a.s. convergence.

Proof. For ' $b 1) \Rightarrow b 2), b 3), c)^{\prime}$ ' see Proposition 3.7 and Remarks 3.3 and 3.10. For ' $c) \Rightarrow b 1$ )' see Remark $3.10 \mathrm{~b}$ ).

'b1) $\Rightarrow a)^{\prime}$ ' will be proved by induction on $n$ :

$n=1: \quad$ Let $\nu \in \mathcal{P}_{\log _{+}^{2}}$. Then the products $\mu=\underset{j \geq 0}{\star} \tau^{j}\left(\nu^{j+1}\right)$ and $\nu_{(1)}=\underset{j \geq 0}{\star} \tau^{j}(\nu)$ are convergent (since $b 1$ ) and hence $\left.b 2\right)$ and $c$ ) hold). Therefore, by Lemma 4.5, $(n=1), \mu=\underset{j \geq 0}{\star} \tau^{j}\left(\underset{i \geq 0}{\star} \tau^{i}(\nu)\right)$. I.e., $\left.a\right)$ follows by Proposition 4.2.

Let ' $b 1) \Rightarrow a$ )' be proved for $k \leq n$. Let $\nu \in \mathcal{P}_{\log _{+}^{n+2}}$, hence $b 2), b 3), c)$ (by Proposition 3.7). b2) yields that $\mu=\underset{k \geq 0}{\star} \tau^{k}\left(\nu^{\left(\begin{array}{c}n+1+k \\ n+1\end{array}\right)}\right)$ 
and $\nu_{(n)}=\underset{i \geq 0}{\star} \tau^{i}\left(\nu^{\left(\begin{array}{c}n+i \\ n\end{array}\right)}\right)$ converge. Using $\sum_{i=0}^{k}\left(\begin{array}{c}n+i \\ n\end{array}\right)=\left(\begin{array}{c}n+1+k \\ n+1\end{array}\right)$, hence $\nu{ }^{\left(\begin{array}{c}n+1+k \\ n+1\end{array}\right)}=\underset{i=0}{\star} \nu{ }^{\left(\begin{array}{c}n+i \\ n\end{array}\right)}$, we obtain (observing that according to c) and Remark 3.3, re-arrangement of convolution products is allowed):

$$
\begin{aligned}
& \mu=\underset{k \geq 0}{\star} \tau^{k}\left(\nu\left(\begin{array}{c}
n+1+k \\
n+1
\end{array}\right)\right)=\underset{k \geq 0}{\star} \underset{i=0}{\star} \underset{i}{k}\left(\nu\left(\begin{array}{c}
n+i \\
n
\end{array}\right)\right) \\
& =\underset{j \geq 0}{\star} \tau^{j}\left(\underset{i \geq j}{\star} \tau^{i}\left(\nu^{\left(\begin{array}{c}
n+i \\
n
\end{array}\right)}\right)\right)=\underset{j \geq 0}{\star} \tau^{j}\left(\nu_{(n)}\right)
\end{aligned}
$$

By Proposition 4.2, $\mu$ is $\tau$-decomposable with - per induction hypothesis $n$-times decomposable - cofactor $\nu_{(n)}$. Hence $a$ ) holds.

If $H_{r}(\mu)=\{e\}$, the converse ' $\left.a\right) \Rightarrow b 2$ )' follows by Lemma 4.5, and $' b 2) \Rightarrow b 1)^{\prime}$ follows by Propositions 3.8 resp. 3.9 .

\section{Multiple $\tau$-Decomposability of CONTinuous CONVOlution SEMIGROUPS AND OF GENERATING FUNCTIONALS}

It is well known that $\tau$-decomposable laws are closely related to limit laws of triangular arrays of random variables of the form $\tau^{k}\left(X_{j}\right)$. However, as already mentioned in the pioneer work [14], even on the real line these arrays are in general not infinitesimal, hence the limits need not be infinitely divisible. Infinitely divisible $\tau$-decomposable laws called $\tau$-semi-self-decomposable - on vector spaces have a considerable rich structure. See e.g., the investigations [16], [17], [15], [18], and the literature mentioned there. Here we discuss briefly the possibility to carry over this concept to the group case.

Throughout we assume again $\mathbb{G}$ to be contractible and $\tau \in \operatorname{Aut}(\mathbb{G})$ to be contracting. If $\mu$ is $\tau$-decomposable and if we assume in addition embeddability of $\mu$ into a continuous convolution semigroup, more generally, into a continuous hemigroup, then there exist infinitesimal triangular arrays converging to $\mu$. We note a slight generalization of [23], 3, Proposition 2:

Proposition 5.1. Let $\tau$ be contracting and $\mu$ be $\tau$-decomposable, $\mu=$ $\nu \star \tau(\mu)$. Assume furthermore that $\nu$ is embeddable into a continuous hemigroup $(\nu(s, t))_{s \leq t}$ of probabilities with $\nu=\nu(0,1)$. Then there exists an uniformly infinitesimal triangular array of the form $\lambda_{i, n}=$ $\tau^{n}\left(\gamma_{i}^{(n)}\right), 1 \leq i \leq k_{n}$, such that

$$
\underset{i=1}{\star k_{n}} \lambda_{i, n}=\tau^{n}\left(\begin{array}{c}
k_{n} \\
\underset{i=1}{\star} \gamma_{i}^{(n)}
\end{array}\right) \stackrel{n \rightarrow \infty}{\rightarrow} \mu
$$

Here $\gamma_{i}^{(n)}$ are probabilities, embeddable into hemigroups for all $n, i$.

Proof. Choose $k_{n} \nearrow \infty$ such that $k_{n+1}-k_{n} \nearrow \infty$ with $k_{0}=0$. E.g., $k_{n}:=n^{2}$. Put $\alpha_{i}:=\nu\left(\frac{i-1-k_{j-1}}{k_{j}-k_{j-1}}, \frac{i-k_{j-1}}{k_{j}-k_{j-1}}\right)$ for $k_{j-1}<i \leq k_{j}, j \geq 0$, and $\gamma_{i}^{(n)}:=\tau^{j-n-1}\left(\alpha_{i}\right)$. $\mu$ is $\tau$-decomposable, hence $\underset{j=0}{\star} \underset{\tau^{j}}{\star}(\nu)=\mu$. 
Therefore,

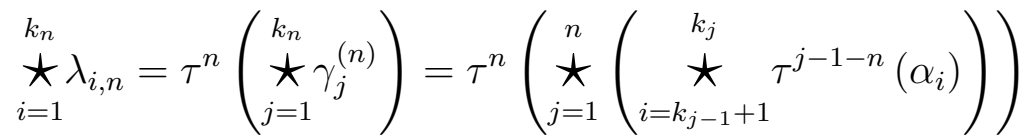

$$
\begin{aligned}
& =\tau^{n}\left(\begin{array}{l}
n \\
\underset{j=1}{\star} \tau^{j-n-1}
\end{array}\left(\begin{array}{cc}
k_{j} & \\
\underset{i=k_{j-1}+1}{\star} & \alpha_{i}
\end{array}\right)\right)=\left(\begin{array}{c}
n \\
\underset{j=1}{\star} \tau^{j-1}
\end{array}\left(\begin{array}{cc}
k_{j} \\
\underset{i=k_{j-1}+1}{\star} & \alpha_{i}
\end{array}\right)\right)
\end{aligned}
$$

By the hemigroup property $\underset{i=k_{j-1}+1}{\star k_{j}} \alpha_{i}=\underset{i=k_{j-1}+1}{\star} \stackrel{k_{j}}{\star} \nu\left(\frac{i-1-k_{j-1}}{k_{j}-k_{j-1}}, \frac{i-k_{j-1}}{k_{j}-k_{j-1}}\right)=$ $\nu(0,1)=\nu$, hence $\underset{i=1}{\star k_{n}} \lambda_{i, n}=\tau^{n}\left(\begin{array}{l}k_{n} \\ \star \\ 1\end{array} \gamma_{j}^{(n)}\right)=\underset{j=0}{\star} \tau^{j}(\nu) \rightarrow \mu$.

To prove infinitesimality, put $\Gamma:=\{\nu(s, t): 0 \leq s \leq t \leq 1\}$. $\Gamma$ is uniformly tight, hence for any neighbourhood $U$ of $e$ and any $\varepsilon>0$ we have $\tau^{n}(\gamma)(\mathbb{G} \backslash U)<\varepsilon$ for sufficiently large $n$, for $n \geq N_{\varepsilon}$ say, and all $\gamma \in \Gamma$.

1. For $n \geq 2 N_{\varepsilon}$ and $j \leq\left[\frac{n}{2}\right]$, hence $n-j \geq N_{\varepsilon}$, if $k_{j-1} \leq i<k_{j}$ then

$$
\tau^{n}\left(\gamma_{i}^{(n)}\right)(\mathbb{G} \backslash U)=\tau^{n-j}\left(\nu\left(\frac{i-1-k_{j-1}}{k_{j}-k_{j-1}}, \frac{i-k_{j-1}}{k_{j}-k_{j-1}}\right)\right)(\mathbb{G} \backslash U)<\varepsilon
$$

2. By continuity of the hemigroup there exists a $\delta>0$ such that for $0 \leq t-s<\delta$ we have $\nu(s, t)(\mathbb{G} \backslash U)<\varepsilon$. By assumption, $k_{n}-k_{n-1} \nearrow \infty$, hence for sufficiently large $n, n \geq L_{\delta}$ say, we have $k_{j}-k_{j-1}>1 / \delta$. For $n \geq 2 L_{\delta}$ and for $j \geq\left[\frac{n}{2}\right]$ we obtain $\nu\left(\frac{i-1-k_{j-1}}{k_{j}-k_{j-1}}, \frac{i-k_{j-1}}{k_{j}-k_{j-1}}\right)(\mathbb{G} \backslash V)<\varepsilon$ for any neighbourhood $V$ of the unit such that $V \subseteq \bigcap_{k \geq 0} \tau^{-k}(U)$. (Cf. e.g., [30], Lemma 1.4, [7], 3.1.3 d) ).

Hence for all $n \geq 2 L_{\delta}$, for all $j \geq\left[\frac{n}{2}\right], k_{j-1} \leq i<k_{j}$ we have

$$
\begin{aligned}
\tau^{n}\left(\gamma_{i}^{(n)}\right)(\mathbb{G} \backslash U) & =\tau^{n-j}\left(\nu\left(\frac{i-1-k_{j-1}}{k_{j}-k_{j-1}}, \frac{i-k_{j-1}}{k_{j}-k_{j-1}}\right)\right)(\mathbb{G} \backslash U) \\
& \leq \nu\left(\frac{i-1-k_{j-1}}{k_{j}-k_{j-1}}, \frac{i-k_{j-1}}{k_{j}-k_{j-1}}\right)(\mathbb{G} \backslash V) \\
& <\varepsilon
\end{aligned}
$$

Whence the assertion follows by 1) and 2).

Definition 5.2. $\mu \in \mathcal{M}^{1}(\mathbb{G})$ is $\tau$-semi-self-decomposable if there exist $\nu_{n} \in \mathcal{M}^{1}(\mathbb{G}), n \in \mathbb{N}$, and $b_{n} \in \operatorname{Aut}(\mathbb{G})$ such that for $k_{n} \rightarrow \infty$ we have

(i) $\quad b_{n}^{-1} b_{n+1} \rightarrow \tau, \quad$ (ii) $b_{n}^{-1}\left(\begin{array}{c}k_{n} \\ * \\ j=1\end{array}\right) \rightarrow \mu$

'*' denoting convolution in revised order, ${ }^{n} \nu_{i}:=\nu_{n} \star \cdots \star \nu_{1}$, and

(iii) The array $\left(\lambda_{n, i}:=b_{n}^{-1}\left(\nu_{j}\right)\right)_{1 \leq i \leq k_{n}}$ is uniformly infinitesimal.

Let $\widetilde{\mathcal{L}}_{s}(\tau)$ denote the set of limits of row products $b_{n}^{-1}\left(\begin{array}{c}k_{n} \\ \star \\ i=0\end{array}\right)$ of arrays satisfying (i) and (ii), and let $\mathcal{L}_{s}(\tau)$ denote the set of $\tau$-semiself-decomposable laws.

We obviously have: 
Proposition 5.3. $\mu \in \widetilde{\mathcal{L}}_{s}(\tau)$ iff $\mu$ is $\tau$-decomposable.

Proof. ' $\Rightarrow$ ' By assumption, $b_{n+1}^{-1} b_{n} \rightarrow \tau$, and $b_{n+1}^{-1}\left(\begin{array}{c}k_{n+1} \\ * \\ j=1\end{array}\right) \rightarrow \mu$.

Hence

$$
\begin{aligned}
& b_{n+1}^{-1}\left(\begin{array}{c}
k_{n+1} \\
\multirow{j}{*}{\nu_{j}} \\
j=1
\end{array}\right)=b_{n+1}^{-1}\left(\begin{array}{cc}
k_{n+1} \\
\multirow{*}{*}{} & \nu_{j} \\
j=k_{n}+1
\end{array}\right) \star\left(b_{n+1}^{-1} b_{n}\right) b_{n}^{-1}\left(\begin{array}{l}
k_{n} \\
* \nu_{i} \\
1
\end{array}\right) \\
& \rightarrow \nu \star \tau(\mu)
\end{aligned}
$$

【 The terms on the left and the second terms on the right hand converge both to $\tau(\mu)$ by assumption, hence the first terms on the right are relatively compact according to the shift compactness theorem, with accumulation point $\nu$. ]

$' \Leftarrow$ ' We have, for any $n$ :

$$
\mu=\lim _{n} \underset{j=0}{\star} \underset{\tau^{n-j}}{n}(\nu)=\lim _{n} \underset{j=0}{*} \underset{j}{*} \tau^{j}(\nu)
$$

The assertion follows with $b_{n}:=\tau^{-n}$ and $\nu_{j}=\tau^{-j}(\nu), 0 \leq j \leq n$.

Obviously the array $\left(\tau^{n}\left(\nu_{j}\right)\right)$ is in general not uniformly infinitesimal.

On vector spaces, limits of uniformly infinitesimal triangular arrays are infinitely divisible, hence embeddable and vice versa. On (nonAbelian) groups embeddability of limits of commutative infinitesimal triangular arrays is only known for particular classes of groups under restrictive conditions. See e.g., [28], [25],[26], [2], [19], [20], [21] and the references mentioned there. In the following we assume again $\mathcal{S} \subseteq \mathcal{M}^{1}(\mathbb{G})$ to be a commutative sub-semigroup with $\tau(\mathcal{S}) \subseteq \mathcal{S}$. If we assume in addition the arrays $\lambda_{n, j}=\left(b_{n}^{-1}\left(\nu_{j}\right)\right)_{1 \leq j \leq k_{n}, n \geq 1}$ to be infinitesimal, under suitable conditions and under the assumption that all measures belong to $\mathcal{S}$, we obtain that $\tau$-semi-self-decomposable laws are embeddable into continuous convolution semigroups.

To motivate the next definitions we consider first the example of real vector spaces:

Example 5.4. Let $\mathbb{G}=\mathbb{R}^{d}, \tau \in \mathrm{GL}\left(\mathbb{R}^{d}\right)$. Let $\mu$ be infinitely divisible, hence embeddable into a uniquely determined continuous convolution semigroup $\left(\mu_{t}\right)_{t \geq 0}$ with $\mu=\mu_{1}$.

1.) If $\mu_{t}$ is $\tau$-decomposable for all $t \geq 0, \mu_{t}=\nu(t) \star \tau\left(\mu_{t}\right)$, then it is easily seen that the cofactors $\{\nu(t): t \geq 0\}$ form a continuous convolution semigroup. Hence we write $\nu(t)=\nu_{t}$. Note that the cofactors are uniquely determined then. « In fact, this follows by uniqueness of embedding and by P. Lévy's uniqueness- and continuity theorem for Fourier transforms on $\mathbb{R}^{d}$.]

2.) Assume that $\mu=\mu_{1}$ is embeddable and $\tau$-decomposable, and moreover, that the cofactor is embeddable. I.e., there exist continuous convolution semigroups $\left(\mu_{t}\right)_{t \geq 0},\left(\nu_{t}\right)_{t \geq 0}$, such that $\mu_{1}=\nu_{1} \star \tau\left(\mu_{1}\right)$. Again, it follows by uniqueness of embedding that $\mu_{t}=\nu_{t} \star \tau\left(\mu_{t}\right)$ for all $t \geq 0$.

3.) Furthermore, if $\mu=\nu \star \tau(\mu)$ and if the cofactor $\nu$ is embeddable into a continuous convolution semigroup $\left(\nu_{t}\right)$ then $\mu$ is embeddable. In 
fact, $\left(\mu_{t}=\lim _{n} \underset{k=0}{\star n} \tau^{k}\left(\nu_{t}\right)\right)_{t \geq 0}$ is a continuous convolution semigroup, since the set of infinitely divisible laws is closed, and $\mu_{1}=\mu$. Hence by 2.), we obtain:

a $\tau$-decomposable law $\mu$ is embeddable iff the cofactor is embeddable.

4.) In case $n=1$, i.e., $\mu_{t}=\nu_{t} \star \tau\left(\mu_{t}\right)$ and $\nu_{t}=\lambda_{t} \star \tau\left(\nu_{t}\right)$ (for all $t$ ), $\mu_{t}=\lambda_{t} \star \tau\left(\nu_{t}\right) \star \tau\left(\mu_{t}\right) \forall t \geq 0$.

Analogously we obtain for $n$-times decomposability (for all $t$ ):

$\mu_{t}=: \nu_{t}^{(n+1)}=\nu_{t}^{(n)} \star \tau\left(\nu_{t}^{(n-1)}\right) \cdots \star \tau\left(\nu_{t}^{(0)}\right)$, and $\nu_{t}^{(i)}=\nu_{t}^{(i-1)} \star \tau\left(\nu_{t}^{(i)}\right)$, $1 \leq i \leq n+1$.

5.) If we consider in 4) Fourier transforms resp. their logarithms, the 'second characteristic functions', we obtain: $\widehat{\nu}_{t}^{(n+1)}=\widehat{\mu}_{t}=\mathrm{e}^{t \cdot L}$, and $\widehat{\nu}_{t}^{(i)}=\mathrm{e}^{t \cdot L^{(i)}}, i=1, \ldots, n+1$, thus $L=L^{(0)}+\sum_{1}^{n+1} L^{(i)} \circ \tau^{*}$, where $L=: L^{(n+1)}, L^{(i)}=L^{(i-1)}+L^{(i)} \circ \tau^{*}, 1 \leq i<n+1$. ( $\tau^{*}$ denoting the adjoint of $\tau \in \mathrm{GL}\left(\mathbb{R}^{d}\right)$.)

This is a motivation for the following definition of (multiple) decomposability of continuous convolution semigroups on groups $\mathbb{G}$. All continuous convolution semigroups are assumed to have trivial idempotents, $\mu_{0}=\varepsilon_{e}$.

Definition 5.5. Let $\mathbb{G}$ be a contractible group with contracting $\tau \in$ $\operatorname{Aut}(\mathbb{G})$.

a) $\left(\mu_{t}\right)_{t \geq 0}$ is called $\tau$-decomposable continuous convolution semigroup if there exists a continuous convolution semigroup of 'cofactors' $\left(\nu_{t}\right)_{t \geq 0}$ such that $\mu_{t}=\nu_{t} \star \tau\left(\mu_{t}\right)$ for all $t \geq 0$. (Note that $\mu_{0}=\varepsilon_{e} \Rightarrow \nu_{0}=\varepsilon_{e}$.)

b) $\left(\mu_{t}\right)_{t \geq 0}$ is n-times $\tau$-decomposable if all $\mu_{t}$ are $\tau$-decomposable and if there exists a continuous convolution semigroup of cofactors $\left(\mu_{t}^{(n)}\right)_{t \geq 0}$, which is $(n-1)$-times $\tau$-decomposable.

c) Recall that continuous convolution semigroups $\left(\mu_{t}\right)$ are identified with their generating functionals $\mathcal{A}=\left.\frac{\mathrm{d}^{+}}{\mathrm{d} t}\right|_{t=0} \mu_{t}$ evaluated at $f \in \mathcal{D}(\mathbb{G})$, the Schwartz-Bruhat function space. Let $\mathcal{G F}(\mathbb{G})$ denote the cone of generating functionals.

In view of $b)$ we define:

$\mathcal{A}=: \mathcal{A}^{(0)} \in \mathcal{G F}(\mathbb{G})$ is $\tau$-decomposable - in symbols, $\mathcal{A} \in \Lambda_{0}(\tau)$ - if $\mathcal{A}=\mathcal{B}+\tau(\mathcal{A})$, for some $\mathcal{B} \in \mathcal{G} \mathcal{F}(\mathbb{G})$.

Put $\Lambda_{-1}:=\mathcal{G F}(\mathbb{G})$ and define analogously for $n \in \mathbb{N}: \mathcal{A}=: \mathcal{A}^{(n+1)}$ is $n$-times $\tau$-decomposable - in symbols, $\mathcal{A} \in \Lambda_{n}(\tau)$ - if $\mathcal{A}$ is $\tau$-decomposable, $\mathcal{A}=\mathcal{A}^{(n)}+\tau(\mathcal{A})$, where $\mathcal{A}^{(n)} \in \Lambda_{n-1}$.

Hence there exist $\mathcal{A}^{(i)} \in \mathcal{G F}(\mathbb{G}), i=1, \ldots, n+1$, such that $\mathcal{A}^{(i)}=$ $\mathcal{A}^{(i-1)}+\tau\left(\mathcal{A}^{(i)}\right)$, therefore $\mathcal{A}=\mathcal{A}^{(0)}+\sum_{i=1}^{n+1} \tau\left(\mathcal{A}^{(i)}\right)$.

Note that c) is in analogy to the vector space case considered in Example 5.4, 4). In fact, generating functionals replace the second characteristic functions. (For more details on continuous convolution semigroups and generating functionals see e.g., [29], [10], [7], [3].) 
Here we used the following notations: For $\mathcal{A} \in \mathcal{G} \mathcal{F}(\mathbb{G})$ and $\sigma \in$ $\operatorname{Aut}(\mathbb{G}), \sigma(\mathcal{A}) \in \mathcal{G} \mathcal{F}(\mathbb{G})$ is defined by $\langle\sigma(\mathcal{A}), f\rangle:=\langle\mathcal{A}, f \circ \sigma\rangle$ for test functions $f \in \mathcal{D}(\mathbb{G})$.

More generally, let $\mathbb{G}_{i}$ be locally compact groups, let $\psi: \mathbb{G}_{1} \rightarrow \mathbb{G}_{2}$ be a homeomorphism which respects the differentiable structure, i.e. $f \circ \psi \in \mathcal{D}\left(\mathbb{G}_{1}\right)$ for all $f \in \mathcal{D}\left(\mathbb{G}_{2}\right)$. Then, $\psi(\mathcal{A}) \in \mathcal{G F}\left(\mathbb{G}_{2}\right)$ for all $\mathcal{A} \in \mathcal{G} \mathcal{F}\left(\mathbb{G}_{1}\right)$. (Where again $\psi(\mathcal{A})$ is defined by $\langle\psi(\mathcal{A}), f\rangle:=\langle\mathcal{A}, f \circ$ $\psi\rangle$ for test functions $f \in \mathcal{D}\left(\mathbb{G}_{2}\right)$.) In fact, $\psi(\mathcal{A}) \in \mathcal{G} \mathcal{F}\left(\mathbb{G}_{2}\right)$ follows immediately by E. Siebert's characterization of generating functionals, [29], Satz 5 , [10], Theorem 4.5.8.

Remark 5.6. As afore mentioned, if $\left(\mu_{t}\right)$ is a continuous convolution semigroup with generating functional $\mathcal{A}$, then $n$-times decomposability of $\left(\mu_{t}\right)$ implies $n$-times decomposability of $\mathcal{A}$. The converse is true if the involved measures $\mu_{t}, \nu_{s}^{(i)}$ belong to a commutative sub-semigroup $\mathcal{S}$ with $\tau(\mathcal{S}) \subseteq \mathcal{S}$.

$n$-times decomposable continuous convolution semigroups and generating functionals (in Lévy Khinchin representation of the second characteristic functions) were investigated for real and $p$-adic vector spaces and characterized in various ways. (Cf. [16], [18].) We use these results to obtain characterizations at least for the main examples, connected resp. totally disconnected contractible groups. First we mention the obvious result:

Lemma 5.7. We assume that as above a homeomorphism $\psi: \mathbb{G}_{1} \rightarrow \mathbb{G}_{2}$ exists respecting the differentiable structures, and in addition there exist $\tau \in \operatorname{Aut}\left(\mathbb{G}_{1}\right), \rho \in \operatorname{Aut}\left(\mathbb{G}_{2}\right)$ such that $\psi \circ \tau=\rho \circ \psi$. Then we have:

a) If $\tau$ is contracting (on $\mathbb{G}_{1}$ ) then so is $\rho$ (on $\mathbb{G}_{2}$ ).

b) If $\mathcal{A} \in \mathcal{G F}\left(\mathbb{G}_{1}\right)$ is (n-times) $\tau$-decomposable then also $\psi(\mathcal{A}) \in$ $\mathcal{G} \mathcal{F}\left(\mathbb{G}_{2}\right)$ is (n-times) $\rho$-decomposable, $n \geq 0$.

The above mentioned results imply Proposition 5.8 below, from which Theorem 1.2 follows. We use the notations introduced in Proposition 2.11. Let $\mathbb{G}$ be a contractible group, $\mathcal{A} \in \mathcal{G F}(\mathbb{G})$ a generating functional.

Proposition 5.8. a) Let $\mathbb{G}$ be a connected contractible locally compact group, i.e., a (homogeneous) Lie group, of dimension d, say. Let $\mathcal{G} \cong \mathbb{R}^{d}$ denote the Lie algebra, exp the exponential mapping and for $\tau \in \operatorname{Aut}(\mathbb{G})$ let $\stackrel{\circ}{\tau} \in \mathrm{GL}(\mathcal{G}) \subseteq \mathrm{GL}\left(\mathbb{R}^{d}\right)$ denote the differential. For $\mathcal{A} \in \mathcal{G} \mathcal{F}(\mathbb{G})$ let $\stackrel{\circ}{\mathcal{A}}:=\exp ^{-1}(\mathcal{A}) \in \mathcal{G} \mathcal{F}(\mathcal{G})$ and $\left(\lambda_{t}\right) \subseteq \mathcal{M}^{1}(\mathcal{G})$ the corresponding continuous convolution semigroup. Then the following assertions are equivalent:

(i) $\mathcal{A}$ is n-times $\tau$-decomposable $($ in $\mathcal{G} \mathcal{F}(\mathbb{G})$ )

(ii) $\stackrel{\circ}{\mathcal{A}}$ is n-times $\stackrel{\circ}{\tau}$-decomposable $\left(\right.$ in $\mathcal{G} \mathcal{F}(\mathcal{G}) \cong \mathcal{G} \mathcal{F}\left(\mathbb{R}^{d}\right)$ )

(iii) The continuous convolution semigroup $\left(\lambda_{t}\right) \subseteq \mathcal{M}^{1}(\mathcal{G})$ generated by $\stackrel{\circ}{\mathcal{A}}$ is n-times $\stackrel{\circ}{\tau}$-decomposable

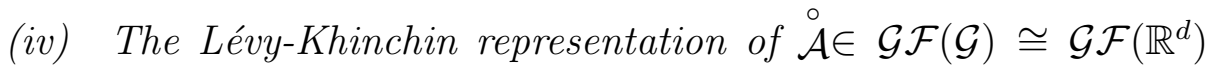
has the structure described in [16], Theorems 3.1. 
b) Let $\mathbb{G}$ be a totally disconnected contractible locally compact group, with contracting $\tau \in \operatorname{Aut}(\mathbb{G})$. (Cf. Examples 2.4, 2.8.) Let $\mathcal{G} \cong \mathbb{Q}_{p}$ denote the tangent object described in Example 2.8, Proposition 2.11 b), with corresponding homeomorphism $\psi: \mathbb{G} \rightarrow \mathbb{Q}_{p}$, and let $\stackrel{\circ}{\tau} \in \operatorname{Aut}\left(\mathbb{Q}_{p}\right)$ such that $\psi \circ \tau=\stackrel{\circ}{\tau} \circ \psi$. For $\mathcal{A} \in \mathcal{G} \mathcal{F}(\mathbb{G})$ let $\stackrel{\circ}{\mathcal{A}}:=\psi(\mathcal{A})$ and $\left(\lambda_{t}\right) \subseteq$ $\mathcal{M}^{1}(\mathcal{G})$ the corresponding continuous convolution semigroup. Then the following assertions are equivalent:

(i) $\mathcal{A}$ is n-times $\tau$-decomposable (in $\mathcal{G} \mathcal{F}(\mathbb{G})$ )

(ii) $\stackrel{\circ}{\mathcal{A}}$ is n-times $\stackrel{\circ}{\tau}$-decomposable $($ in $\mathcal{G} \mathcal{F}(\mathcal{G})$ )

(iii) The continuous convolution semigroup $\left(\lambda_{t}\right) \subseteq \mathcal{M}^{1}(\mathcal{G})$ generated by $\stackrel{\circ}{\mathcal{A}}$ is n-times $\stackrel{\circ}{\tau}$-decomposable

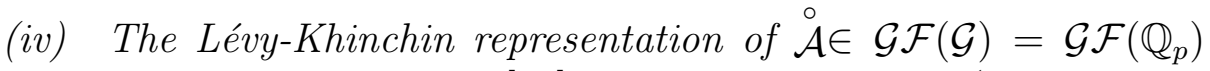
has the structure described in [18], Theorems 4.3, 5.2. (Note that on totally disconnected groups generating functionals are defined by the Lévy measures, which are characterized in [18].)

Proof. a) $\psi:=\exp ^{-1}: \mathbb{G} \rightarrow \mathcal{G} \cong \mathbb{R}^{d}$ satisfies the assumptions of the Lemma 5.7 since $\mathbb{G}$ is a simply connected nilpotent Lie group. The equivalences of $(i) \Leftrightarrow(i i)$ resp. (ii) $\Leftrightarrow$ (iii) follow by Lemma 5.7 b) resp. Remark 5.6, whereas ' $(i i i) \Leftrightarrow(i v)$ ' is proved in [16], Theorem 3.1.

b) By Example 2.8 and Lemma 5.7 the equivalences $(i)-(i i i)$ follow as in a). The equivalence $(i i i) \Leftrightarrow(i v)$ follows from [18], Theorems 4.3, 5.2. [ In fact, in [18] the result is proved for vector spaces over the field of $p$-adic numbers, hence for prime powers $p$. But, at least for dimension 1 , the proof also works for the additive group of the ring of $p$-adic numbers, $p$ not necessarily a prime power.

\section{Appendix: Multiple Decomposability on matrix-CONE HYPERGROUPS}

The investigations before are not limited to the case of groups. If we stay in the locally compact set-up, hypergroups are natural candidates. For the background the reader is referred to the monograph of W. Bloom and H. Heyer [1]. Recently M. Voit [34] and M. Rösler [24] investigated new classes of hypergroup structures on matrix cones with 'group like' properties. In [5] some basic probabilistic aspects of these hypergroups were investigated. It turned out that most of the tools needed for the investigations above in Sections 3-5 are also available for these hypergroups: There exist norms and automorphisms, in particular, contractible automorphisms. Furthermore, shift compactness theorems ([5], 0.12, 1.10), and a version of the equivalence theorem ([5], 2.20) are proved. Moreover, the convolution is commutative and Hermitean, hence by Lévy's continuity theorem for hypergroups convergence of infinite convolution products and triangular arrays are easier to handle. $\tau$-decomposable laws (the case $n=0$ ) and their co-factors are characterized ([5], 2.21-2.23). Altogether, most of the results for multiple decomposable laws obtained before have a counterpart (at 
least on) on matrix cone hypergroups. We do not go into details here. The investigations will be continued and published else where.

\section{REFERENCES}

[1] Bloom, W., Heyer, H.: Harmonic Analysis of Probability Measures on Hypergroups. Walter de Gruyter, Berlin-New York (1995)

[2] Guivarc'h, Y., Shah, R.: Asymptotic properties of convolution operators and limit distributions of triangular arrays on locally compact groups. Trans. Amer. Math. Soc. 357, 3683-3729 (2005)

[3] Hazod, W.: Stetige Faltungshalbgruppen von Wahrscheinlichkeitsmaßen und erzeugende Distributionen. Lecture Notes in Mathematics 595. Springer (1977)

[4] Hazod, W.: Multiple selfdecomposable laws on vector spaces and on groups: The existence of background driving processes. In: Statistical Inference, Economic Analysis and Matrix Algebra. B. Schipp, W. Krämer ed. 295-307. Physica Verlag (2008)

[5] Hazod, W.: Probability on matrix-cone hypergroups: Limit theorems and structural properties. J. Appl. Anal. 15, 205-245 (2009)

[6] Hazod, W., Scheffler, H-P.: Strongly $\tau$-decomposable and selfdecomposable laws on simply connected nilpotent groups. Monatsh. Math. 128, 269-282 (1999)

[7] Hazod, W., Siebert, E.: Stable Probability Measures on Euclidean Spaces and on Locally Compact Groups. Structural Properties and Limit Theorems. Mathematics and its Applications vol. 531. Kluwer A.P. (2001)

[8] Hebisch, W., Sikora, A.: A smooths subadditive homogeneous norm on a homogeneous group. Stidia Math. 96, 231-236 (1990)

[9] Hewitt, E., Ross, K.A.: Abstract Harmonic Analysis I. Berlin-HeidelbergNew York, Springer (1970)

[10] Heyer, H.: Probability Measures on Locally Compact Groups. Berlin-Heidelberg-New York, Springer (1977)

[11] Jurek, Z., Mason, D.: Operator Limit Distributions in Probability Theory. J. Wiley Inc. (1993).

[12] Krakowiak, W.: Zero-one-laws for A-decomposable measures on Banach spaces. Bull. Pol. Acad. Sci. Math. 33, 85-90 (1985)

[13] Kosfeld, K.: Zerlegbarkeitseigenschaften von Verteilungen auf lokalkompakten Gruppen. Dissertation, Technische Universität Dortmund (2009). Electronic version available: http://hdl.handle.net/2003/27013

[14] Loève, M.: Nouvelles classes de lois limites. Bull. Soc. Math. Fr. 73, 107-206 (1945)

[15] Maejima, M., Naito, Y.: Semi-selfdecomposable distributions and a new class of limit laws. Probab. Theory Related Fields 112, 13-31 (1998)

[16] Maejima, M., Sato, K.I., Watanabe, T.: Operator semi-selfdecomposability, (C,Q)-decomposability and related nested classes. Tokyo J. Math. 22, 473-509 (1999)

[17] Maejima, M., Sato, K.I., Watanabe, T.: Completely operator semi-selfdecomposable distributions. Tokyo J. Math. 23, 235-253 (2000)

[18] Maejima, M., Shah, R.: Operator semistable, operator semi-selfdecomposable probability measures and related nested classes on $p$-adic vector spaces. Monatsh. Math. 151, 293-318 (2007)

[19] Neuenschwander, D.: Limits of commutative triangular systems on simply connected step 2-nilpotent Lie groups. J. Theoret. Probab. 5, 217-222 (1992)

[20] Neuenschwander, D.: Triangular systems on discrete subgroups of simply connected nilpotent Lie groups. Publ. Math. Debrecen 47, 329-333 (1995)

[21] Neuenschwander, D.: Triangular systems of probability measures on simply connected nilpotent Lie groups and discrete subgroups of exponential Lie groups. C.R. Acad. Sci. Paris 333, 1029-1034 (2001)

[22] Parthasarathy, K.R.: Probability measures on metric spaces. Academic Press (1967) 
[23] Raja, C.R.E.: Operator semi-selfdecomposable probability measures and related nested subclasses of measures on vector spaces. Monatsh. Math. 142, 351-361 (1992)

[24] Rösler, M.: Bessel convolutions on matrix cones. Compos. Math. 143, 749779 (2007)

[25] Shah, R.: Limits of commutative triangular systems on real and p-adic groups. Math. Proc. Camb. Phil. Soc. 120, 181-192 (1996)

[26] Shah, R.: The central limit theorem on locally compact groups. Israel J. Math. 110, 189-218 (1999)

[27] Shah, R.: Selfdecomposable measures on simply connected nilpotent Lie groups. J. Theoret. Probab. 13, 65-83 (2000)

[28] Shah, R.: Limits of commutative triangular systems on locally compact groups. Proc. Indian Math. Soc. 111, 49-63 (2001)

[29] Siebert, E.: Über die Erzeugung von Faltungshalbgruppen auf beliebigen lokalkompakten Gruppen. Math. Z. 131, 313-333 (1973)

[30] Siebert, E.: Contractive automorphisms on locally compact groups. Math. Z. 191, 73-90 (1986)

[31] Siebert, E.: Strongly operator-decomposable probability measures on separable Banach spaces. Math. Nachr. 154, 315-326 (1991)

[32] Siebert, E.: Operator decomposability of Gaussian measures on separable Banach spaces. J. Theoret. Probab. 5, 333-347 (1992)

[33] Tkačenko, M.: Introduction to topological groups. Topology Appl. 86, 179231 (1998)

[34] Voit, M.: Bessel convolutions on matrix cones: Algebraic properties and random walks. J. Theoret. Probab. 22, 741-771 (2009)

[35] Wang, J.S.P.: The Mautner pheneomen on p-adic Lie groups. Math. Z. 185, 403-412 (1984)

Faculty of Mathematics, Technische Universität Dortmund, D-44221 DorTMund, GERMANY

E-mail address: wilfried.hazod@math.uni-dortmund.de

E-mail address: Katrin.Kosfeld@math.uni-dortmund.de 


\section{Preprints ab 2009/04}

2011-04

2011-03

2011-02

2011-01

2010-16

2010-15

2010-14

2010-13

2010-12

2010-11

2010-10

2010-09

2010-08

2010-07

2010-06

2010-05

2010-04
Wilfried Hazod, Katrin Kosfeld

Multiple decomposability of probabilities on contractible locally compact groups

Alexandra Monzner* and Frol Zapolsky†

A comparison of symplectic homogenization and Calabi quasi-states

Stefan Jäschke, Karl Friedrich Siburg and Pavel A. Stoimenov

Modelling dependence of extreme events in energy markets using tail copulas

Ben Schweizer and Marco Veneroni

The needle problem approach to non-periodic homogenization

Sebastian Engelke and Jeannette H.C. Woerner

A unifying approach to fractional Lévy processes

Alexander Schnurr and Jeannette H.C. Woerner

Well-balanced Lévy Driven Ornstein-Uhlenbeck Processes

Lorenz J. Schwachhöfer

On the Solvability of the Transvection group of Extrinsic

Symplectic Symmetric Spaces

Marco Veneroni

Stochastic homogenization of subdifferential inclusions via scale integration

Agnes Lamacz, Andreas Rätz, and Ben Schweizer

A well-posed hysteresis model for flows in porous media and applications to fingering effects

Luca Lussardi and Annibale Magni

$\Gamma$-limits of convolution functionals

Patrick W. Dondl, Luca Mugnai, and Matthias Röger

Confined elastic curves

Matthias Röger and Hendrik Weber

Tightness for a stochastic Allen-Cahn equation

Michael Voit

Multidimensional Heisenberg convolutions and product formulas

for multivariate Laguerre polynomials

Ben Schweizer

Instability of gravity wetting fronts for Richards equations with hysteresis

Lorenz J. Schwachhöfer

Holonomy Groups and Algebras

Agnes Lamacz

Dispersive effective models for waves in heterogeneous media

Ben Schweizer and Marco Veneroni

Periodic homogenization of Prandtl-Reuss plasticity equations in arbitrary dimension 
2010-02 René L. Schilling and Alexander Schnurr

The Symbol Associated with the Solution of a Stochastic Differential Equation

2010-01 Henryk Zähle

Rates of almost sure convergence of plug-in estimates for distortion risk measures

2009-16 Lorenz J. Schwachhöfer

Nonnegative curvature on disk bundles

2009-15 Iuliu Pop and Ben Schweizer

Regularization schemes for degenerate Richards equations and outflow conditions

2009-14 Guy Bouchitté and Ben Schweizer

Cloaking of small objects by anomalous localized resonance

2009-13 Tom Krantz, Lorenz J. Schwachhöfer

Extrinsically Immersed Symplectic Symmetric Spaces

2009-12 Alexander Kaplun

Continuous time Ehrenfest process in term structure modelling

2009-11 Henryk Zähle

Ein aktuarielles Modell für die Portabilität der Alterungsrückstellungen in der PKV

2009-10 Andreas Neuenkirch and Henryk Zähle

Asymptotic error distribution of the Euler method for SDEs with non-Lipschitz coefficients

2009-09 Karl Friedrich Siburg, Pavel A. Stoimenov

Regression dependence

2009-08 Wilfried Hazod

Continuous convolution hemigroups integrating a sub-multiplicative function

2009-07 Sergio Conti and Ben Schweizer

On optimal metrics preventing mass transfer

2009-06 Simon Castle, Norbert Peyerimhoff, Karl Friedrich Siburg

Billiards in ideal hyperbolic polygons

2009-05 Ludwig Danzer

Quasiperiodic Tilings - Substitution Versus Inflation

2009-04 Flavius Guiaş

Direct simulation of the infinitesimal dynamics of semi-discrete approximations for convection-diffusion-reaction problems 